\title{
Nonlinearities in Stereoscopic Phase-Differencing
}

\author{
James Peter Monaco, Member, IEEE, Alan Conrad Bovik, Fellow, IEEE, and Lawrence K. Cormack
}

\begin{abstract}
Exploiting the quasi-linear relationship between local phase and disparity, phase-differencing registration algorithms provide a fast, powerful means for disparity estimation. Unfortunately, these phase-differencing techniques suffer a significant impediment: phase nonlinearities. In regions of phase nonlinearity, the signals under consideration possess properties that invalidate the use of phase for disparity estimation. This paper uses the amenable properties of Gaussian white noise images to analytically quantify these properties. The improved understanding gained from this analysis enables us to better understand current methodologies for detecting regions of phase instability. Most importantly, we introduce a new, more effective means for identifying these regions based on the second derivative of phase.
\end{abstract}

Index Terms-Disparity estimation, Gabor functions, gaussian random processes, instantaneous frequency, local correlation, local phase, stereopsis.

\section{INTRODUCTION}

A $\mathrm{S}$ an alternative to typical feature based algorithms, phase-based techniques, such as phase-differencing, have proven to be a powerful means for stereo image correspondence [1]-[15]. These techniques can produce disparity maps to sub-pixel accuracy, without requiring explicit sub-pixel reconstruction. Since relevant phase information is available at any point in the image, not only at specific tokens as in feature based methods [8], [16], phase-based methods yield dense disparity maps. Additionally, phase is relatively insensitive to typical interimage differences such as lighting, shadows, and noise [17]. Unfortunately, phase-differencing techniques suffer from a significant impediment: phase nonlinearities.

The premise behind phase-differencing methods originates in the Fourier shift theorem, which states that the Fourier transforms of two signals related by a global shift are themselves identical up to a phase difference equal to the frequency times the shift. This linear relationship makes it possible to immediately recover this shift or disparity at any frequency (that is present in the signal) by simply dividing the phase difference by the frequency. Unfortunately, stereo images are not related by a global shift, but instead, are better modeled by many local shifts. Consequently, the generation of accurate disparity maps requires a spatially localized estimate of the phase. Such localization is often accomplished by windowing the complex exponential function. The introduction of this windowing func-

Manuscript received August 17, 2006; revised May 21, 2008. Published August 13, 2008 (projected). This work was supported in part by the National Science Foundation under Grant 0427372 . The associate editor coordinating the review of this manuscript and approving it for publication was Dr. Hassan Foroosh.

J. P. Monaco and A. C. Bovik are with the Department of Electrical and Computer Engineering, The University of Texas at Austin, Austin TX 78712-1084 USA (e-mail: monaco@ece.utexas.edu; bovik@ece.utexas.edu).

L. K. Cormack is with the Department of Psychology, The University of Texas at Austin, Austin TX 78712-0187 USA.

Digital Object Identifier 10.1109/TIP.2008.2001405 tion disrupts the simple, linear relation between phase and disparity. In order to successfully apply phase-differencing to a pair of stereo images it is necessary to completely understand these disruptive effects. Perhaps the most comprehensive paper in this direction is [18]. Here, Fleet et al. models images as 1-D Gaussian white noise processes and derives bounds for the expected mean phase difference and absolute mean phase difference under the operations of translation and dilation. In [19], Cai considers the instability of localized phase from the viewpoint that registration by phase-differencing can be considered as a type of Newton iteration. He points out that the instability of phase measurements in certain regions violates the criteria necessary for convergence.

A key to successfully applying phase-differencing stereo reconstruction algorithms lies in the identification of regions of phase instability, i.e., the portions of signals that possess specific properties that invalidate the use of phase for disparity estimation. Several papers address this issue [10], [18], [20], [21], focusing primarily on the same two means for instability detection. Though these techniques have been shown to be empirically successful, their foundations are somewhat heuristic and their efficacy has never been fully examined analytically.

One intent of this work is to supplement the existing literature by filling in some important gaps in the current understanding of localized phase for disparity estimation. Modeling the images as Gaussian white noise processes, we analytically quantify the behavior of the the first and second derivatives of local phase. The first derivative describes the linear relation between phase and disparity. The second derivative provides valuable information about the nonlinearity of localized phase. We demonstrate that the current methods for identifying regions of instability are simply approximations of the second derivative. Finally, we use the information inherent in the second derivative to create a new, more effective means for detecting these regions of nonlinearity.

\section{PhASE-Differencing FOR STEREO CORRESPONDENCE}

In this section, we first present the phase-differencing algorithm and review its underlying theory. Next, we discuss conditions under which phase-differencing is inappropriate. We then offer a new paradigm for identifying these conditions and demonstrate how previous methods of identification that are prominent in the literature are better understood in light of this paradigm.

\section{A. Review}

Given a pair of stereo images $i_{1}(x)$ and $i_{2}(x)$, the goal of a stereo correspondence algorithm is to match each point in $i_{1}(x)$ with its corresponding point in $i_{2}(x)$. If the point $x_{o}$ in $i_{1}(x)$ corresponds to the point $x_{o}-\delta$ in $i_{2}(x)$ then $\delta$ is said to be the disparity relating $i_{1}(x)$ to $i_{2}(x)$ at $x_{o}$.

The methodology used for recovering disparity by phase-differencing is simple. Each image is convolved with a complex 
kernel $h(x)$ whose real and imaginary parts are Hilbert Transforms of one another (i.e., quadrature filters). For our purposes, we assume the filter is formed by windowing the complex exponential with a real, symmetric function as follows: $h(x)=g(x) e^{j \omega_{0} x}$. The windowing function $g(x)$ must be chosen appropriately such that real and imaginary parts of $h(x)$ remain Hilbert transforms of each other. If $O_{1}(x)$ and $O_{2}(x)$ represent the convolutions of $i_{1}(x)$ and $i_{2}(x)$ with $h(x)$, then the disparity is estimated with a single calculation: $\delta(x)=\Delta \theta(x) / \omega_{0}$, where $\Delta \theta(x)=\arg \left[O_{2}(x)\right]-\arg \left[O_{1}(x)\right]$ is the difference in phase between the two responses.

For simplicity, let us only consider the responses at the point $x_{o}=0$. This allows us to replace the convolution with the inner product. Accordingly, we now have $O_{j}=\left\langle i_{j}(x), g(x) e^{j \omega_{0} x}\right\rangle$, where $\left\langle f_{1}, f_{2}\right\rangle=\int f_{1}^{*} f_{2} d x$. The equation to recover disparity becomes

$$
\delta=\frac{\Delta \theta}{\omega_{0}}
$$

which is no longer a function of the positional variable $x$.

In order to better understand the principles governing phasedifferencing, consider the simple case where the windowing function $g(x)=1$ and the images $i_{1}(x)$ and $i_{2}(x)$ are related by a single, global translation, i.e., $i_{2}(x)=i_{1}(x-\delta)$. Now, we have $O_{1}=I\left(-\omega_{0}\right)$ and $O_{2}=I\left(-\omega_{0}\right) e^{j \omega_{0} \delta}$, where $I(\omega)$ is the Fourier transform of $i(x)$. Furthermore, $\Delta \theta=\arg \left[O_{2}\right]-$ $\arg \left[O_{1}\right]=\omega_{o} \delta$, and $\delta$ can be recovered using (1). For complex exponentials the relationship between phase and translation is linear with the relational constant $\omega_{o}$.

Since actual stereo images are not generally related by a single global shift, but instead, by many local shifts (and other deformations), the window $g(x)=1$ with its infinite spatial extent is not very useful. The apparent solution would seem to be the localization of the window. Unfortunately, there is no guarantee that disparity will remain a linear function of phase. That is, (1) may no longer hold. Fortunately, it turns out that phase is linear except in limited regions of phase instability. The identification of these regions of nonlinear phase is the topic of Section II-B.

\section{B. Regions of Phase Nonlinearity}

In this section, we discuss the spatial localization of the window $g(x)$ and its effects on the linearity of phase. We begin by assuming that within the window's spatial extent, defined as the region containing nearly all its energy, the functions $i_{1}(x)$ and $i_{2}(x)$ are modeled reasonably well by a single translation $\delta$, i.e., $i_{2}(x)=i_{1}(x-\delta)$ within $g(x)$. Let us now express $O$ as a function of $\delta: O(\delta)=\left\langle i(x-\delta), g(x) e^{j \omega_{0} x}\right\rangle$, $O_{1}=O(0)$, and $O_{2}=O(\delta)$. Henceforth, we will refer only to $i$ and $O$ and not $i_{1}, i_{2}, O_{1}$, and $O_{2}$. Note that a translation of the image is equivalent to an opposite translation of the filter since $\left\langle i(x-\delta), g(x) e^{j \omega_{o} x}\right\rangle=\left\langle i(x), g(x+\delta) e^{j \omega_{o}(x+\delta)}\right\rangle$. Since local phase is analytic everywhere except at the zeros of $O$, we can use this relation to expand $\Delta \theta$ into a Taylor series

$$
\begin{aligned}
\Delta \theta & =\arg [O(\delta)]-\arg [O(0)] \\
& =\tilde{\omega} \delta+\frac{1}{2 \tilde{\omega}^{\prime} \delta^{2}}+\bigcirc\left(\delta^{3}\right)
\end{aligned}
$$

where the instantaneous frequency $\tilde{\omega}$ is the constant of linearity and its derivative $\tilde{\omega}^{\prime}$ indicates the degree of nonlinearity. If the phase difference is dominated by the linear term $\tilde{\omega}$, the disparity can be recovered in a single step by using this modified version of (1)

$$
\delta=\frac{\Delta \theta}{\tilde{\omega}} .
$$

Two situations arise that may prevent the accurate recovery of phase from (3). First, the phase difference may not be linearly related to the disparity, i.e., the higher order terms of (2) may dominate the linear term. Second, the relationship may be linear, but $\tilde{\omega}$ may be so large as to preclude the detection of disparities beyond a maximum value due to phase wrapping. Phase wrapping forces $|\Delta \theta|<\pi$, and, consequently, $|\delta|<\pi / \tilde{\omega}$ from (3).

The literature proposes the use of two constraints to avoid these areas of phase instability (or detect them by identifying regions where the constraints are violated). The first constraint requires that $\tilde{\omega}$ not deviate too far from the center frequency $\omega_{o}$ [11], [12]

$$
\left|\tilde{\omega}-\omega_{o}\right|<\rho_{1} .
$$

This requirement is often given in the context of detecting phase instability due to scaling deformations between views, but we will see it is also very valuable for nonlinearities due to translation. The simultaneous use of a second constraint was proposed by Fleet [22]. This second constraint requires

$$
\frac{\left.|| O\right|_{x} \mid}{|O|}<\rho_{2}
$$

where $|O|=\sqrt{\operatorname{Re}[O]^{2}+\operatorname{Im}[O]^{2}}$ and $|O|_{x}$ is the partial derivative of $|O|$ with respect to $x$. This requirement is a consequence of the instability of phase near singularities, i.e., points where $|O|=0$ and phase is undefined. The first-order estimate of the distance to such a point is $|O| /\left.|| O\right|_{x} \mid$. Inverting this ratio we get (5).

In [18], Fleet proposed combing the constraints

$$
\sqrt{\left(\tilde{\omega}-\omega_{0}\right)^{2}+\left(\frac{|O|_{x}}{|O|}\right)^{2}}<\rho_{3} .
$$

Whereas the previous constraint (5) approximated the spatial distance from the point of evaluation to a singularity, this combined constraint approximates the scale-space distance (i.e., using both space and scale) to the singularity [23]. Later, we will further substantiate that this combination is more appropriate than employing each constraint independently. This claim was indirectly asserted by Cai [19] when he demonstrated that the region of convergence of a Newton iteration using (3) is the disk bounded by (6).

To simplify further discussions we present the following definitions:

$$
\begin{aligned}
& a+j b=\left\langle i(x), g(x) e^{j \omega_{0} x}\right\rangle \\
& c+j d=\left\langle i(x), g^{\prime}(x) e^{j \omega_{0} x}\right\rangle \\
& e+j f=\left\langle i(x), g^{\prime \prime}(x) e^{j \omega_{0} x}\right\rangle
\end{aligned}
$$


where, for example, $a=\int i(x) \cos \left(\omega_{o} x\right) g(x) d x$. Using these definitions we can express $\theta, \tilde{\omega}$, and $\tilde{\omega}^{\prime}$ as follows:

$$
\begin{aligned}
\theta & =\tan ^{-1}\left(\frac{b}{a}\right) \\
\tilde{\omega} & =\frac{\partial}{\partial x}\left[\tan ^{-1}\left(\frac{b}{a}\right)\right]=\omega_{o}+\frac{a d-b c}{a^{2}+b^{2}}=\omega_{o}+\xi \\
\tilde{\omega}^{\prime} & =\frac{\partial}{\partial x}\left[\omega_{o}+\frac{a d-b c}{a^{2}+b^{2}}\right]=\frac{a f-b e}{a^{2}+b^{2}}-2 \frac{a d-b c}{a^{2}+b^{2}} \frac{a c+b d}{a^{2}+b^{2}} \\
& =\tau-2 \xi \chi=\tau-2 \nu .
\end{aligned}
$$

The representation of $\tilde{\omega}$ was first noted by Papoulis [24]. The constraints presented in (4), (5), and (6) can also be reprised using (7)

$$
\begin{aligned}
\left|\tilde{\omega}-\omega_{o}\right| & =|\xi|<\rho_{1} \\
\frac{\left.|| O\right|_{x} \mid}{|O|} & =\left|\frac{a c+b d}{a^{2}+b^{2}}\right|=|\chi|<\rho_{2} \\
\sqrt{\left(\tilde{\omega}-\omega_{o}\right)^{2}+\left(\frac{|O|_{x}}{|O|}\right)^{2}} & =\sqrt{\xi^{2}+\chi^{2}}<\rho_{3} .
\end{aligned}
$$

Interestingly, the linear term $\tilde{\omega}$ in (9) consists of $\omega_{o}$, corresponding to the center frequency of the complex exponential, and the additional term $\xi$ induced by the windowing function. We should also note that $\xi$ is precisely the term constrained in (11). More importantly, $\tilde{\omega}^{\prime}$, the term in (2) that most directly indicates the degree of nonlinearity, contains the two most prominently advocated constraints in detecting regions of phase instability, $\xi$ and $\chi$. By limiting the ranges of these variables, the possible values of $\tilde{\omega}^{\prime}$ are concomitantly restricted. We will delve into these issues at greater depth in Sections III and IV.

\section{GAUSSIAN RANDOM IMAGES}

In this section, we quantify the effect that the spatial localization of $g(x)$ has on the linear relation between phase and disparity. In order to produce analytic results, the image $i(x)$ will be modeled as the stationary Gaussian white noise process $\boldsymbol{i}(x)$. The nice properties of Gaussian random variables (GRVs) will allow us to analytically derive simple, closed-form results for many important properties of localized phase. We should note that white noise can not model all aspects of real images. Phenomena such as occlusions, foreshortening, and shadowing are disregarded. Furthermore, white noise is spread spectrum, while real images tend to concentrate their energy near DC.

Since $\boldsymbol{i}(x)$ is a Gaussian random process, the integrals in (7) become GRVs. In Sections III-A-D, we first determine the means, variances, and covariances of these variables. We then derive relevant distributions and moments that will help us better understand $\tilde{\omega}$ and $\tilde{\omega}^{\prime}$ and their importance to linear phase. Finally, we quantify the effects of the constraints in (11)-(13) in relation to these distributions and moments.

\section{A. Means, Variances, and Covariances}

In this section, we explore the mean, variances, and covariances of the the random variables that result from (7) when $\boldsymbol{i}(x)$ is a white noise process. The random variables $\boldsymbol{a}, \boldsymbol{b}, \boldsymbol{c}, \boldsymbol{d}, \boldsymbol{e}$, and $\boldsymbol{f}$ are all zero mean due to the zero-mean ergodicity of $i(x)$. Since the kernel $g(x) e^{j \omega_{o} x}$ is defined to be a quadrature filter (its real and imaginary components are Hilbert transforms of each other) the variances become

$$
\begin{aligned}
\sigma_{a}^{2} & =\mathrm{E}\left[\boldsymbol{a}^{2}\right]=\int\left[\cos \left(\omega_{o} x\right) g(x)\right]^{2} d x \\
& =\int\left[\sin \left(\omega_{o} x\right) g(x)\right]^{2} d x=\mathrm{E}\left[\boldsymbol{b}^{2}\right]=\sigma_{b}^{2} \\
\sigma_{c}^{2} & =\mathrm{E}\left[\boldsymbol{c}^{2}\right]=\int\left[\cos \left(\omega_{o} x\right) g^{\prime}(x)\right]^{2} d x \\
& =\int\left[\sin \left(\omega_{o} x\right) g^{\prime}(x)\right]^{2} d x=\mathrm{E}\left[\boldsymbol{d}^{2}\right]=\sigma_{d}^{2} \\
\sigma_{e}^{2} & =\mathrm{E}\left[\boldsymbol{e}^{2}\right]=\int\left[\cos \left(\omega_{o} x\right) g^{\prime \prime}(x)\right]^{2} d x= \\
& =\int\left[\sin \left(\omega_{o} x\right) g^{\prime \prime}(x)\right]^{2} d x=\mathrm{E}\left[\boldsymbol{f}^{2}\right]=\sigma_{f}^{2} .
\end{aligned}
$$

Furthermore, the orthogonality between the real and imaginary parts of quadrature filters necessitates that $\mathrm{E}[\boldsymbol{a b}]=0$, $\mathrm{E}[\boldsymbol{c d}]=0$, and $\mathrm{E}[\boldsymbol{e f}]=0$. In fact, the variables $\boldsymbol{a}, \boldsymbol{b}, \boldsymbol{c}$, and $\boldsymbol{d}$ are all uncorrelated (and, hence, independent). As proof, consider the following:

$$
\begin{aligned}
\mathrm{E}[\boldsymbol{a d}] & =\frac{\omega_{o}}{2} \int\left[\sin ^{2}\left(\omega_{o} x\right)-\cos ^{2}\left(\omega_{o} x\right)\right] g^{2}(x) d x \\
& =\frac{\omega_{o}}{2}\left(\mathrm{E}\left[\boldsymbol{b}^{2}\right]-\mathrm{E}\left[\boldsymbol{a}^{2}\right]\right)=0 .
\end{aligned}
$$

Verifying that the remaining combinations are also zero requires either following the previous derivation or noticing that the integrands are odd functions.

Some cross correlations involving the random variables $e$ and $f$ are generally nonzero

$$
\begin{aligned}
\mathrm{E}[\boldsymbol{a e}] & =\int g^{\prime \prime}(x) g(x) \cos ^{2}\left(\omega_{o} x\right) d x \\
& =2 \omega_{o} \cdot \mathrm{E}[\boldsymbol{a d}]-\mathrm{E}\left[\boldsymbol{d}^{2}\right]=-\sigma_{c}^{2} \\
\mathrm{E}[\boldsymbol{b} \boldsymbol{f}] & =\int g^{\prime \prime}(x) g(x) \sin ^{2}\left(\omega_{o} x\right) d x \\
& =-2 \omega_{o} \cdot \mathrm{E}[\boldsymbol{a d}]-\mathrm{E}\left[\boldsymbol{c}^{2}\right]=-\sigma_{c}^{2} .
\end{aligned}
$$

Equations (18) and (19) follow from integration by parts and then substitution using (17). The remaining cross correlations are zero. Their integrands either form odd functions or follow the derivation as follows:

$$
\begin{aligned}
\mathrm{E}[\boldsymbol{c} \boldsymbol{f}] & =\frac{\omega_{o}}{2} \int g^{\prime \prime}(x) g^{\prime}(x) \cos \left(\omega_{o} x\right) \sin \left(\omega_{o} x\right) d x \\
& =\frac{\omega_{o}}{2}\left(\mathrm{E}\left[\boldsymbol{c}^{2}\right]-\mathrm{E}\left[\boldsymbol{d}^{2}\right]\right)=0 .
\end{aligned}
$$

In summary, the covariance matrix $K$ relating $\boldsymbol{a}, \boldsymbol{c}$, and $\boldsymbol{e}$ is identical to the covariance matrix relating $\boldsymbol{b}, \boldsymbol{d}$, and $\boldsymbol{f}$

$$
K=\left[\begin{array}{ccc}
\sigma_{a}^{2} & 0 & -\sigma_{c}^{2} \\
0 & \sigma_{c}^{2} & 0 \\
-\sigma_{c}^{2} & 0 & \sigma_{e}^{2}
\end{array}\right]=\left[\begin{array}{ccc}
\sigma_{b}^{2} & 0 & -\sigma_{d}^{2} \\
0 & \sigma_{d}^{2} & 0 \\
-\sigma_{d}^{2} & 0 & \sigma_{f}^{2}
\end{array}\right] .
$$

The random variables $\boldsymbol{a}, \boldsymbol{c}$, and $\boldsymbol{e}$ are independent of $\boldsymbol{b}, \boldsymbol{d}$, and $\boldsymbol{f}$.

\section{B. Instantaneous Frequency}

The instantaneous frequency $\tilde{\omega}$ is expressed as the sum of two terms in (9). The first term $\omega_{0}$ represents the center frequency of the bandpass filter $g(x) e^{j \omega_{0} x}$ and corresponds to the constant 
change in phase caused by a global shift in $i(x)$ when the windowing function $g(x)$ is constant and of infinite support. Localizing $e^{j \omega_{o} x}$ with $g(x)$ induces the second term $\xi$. The random variable $\boldsymbol{\xi}$ corresponding to this localization term is a function of the four independent, zero-mean, Gaussian random variables $a, b, c$, and $d$. Its distribution (see Appendix A) is

$$
f(\xi)=\frac{\frac{\sigma_{c}^{2}}{\sigma_{a}^{2}}}{2\left(\frac{\sigma_{c}^{2}}{\sigma_{a}^{2}+\xi^{2}}\right)^{3 / 2}} .
$$

The random variable $\boldsymbol{\xi}$ is zero mean with infinite variance. The absolute deviation exists

$$
\mathrm{E}[|\xi|]=\sqrt{\frac{\sigma_{c}^{2}}{\sigma_{a}^{2}}} .
$$

\section{Derivative of the Instantaneous Frequency}

Analysis of the derivative of the instantaneous frequency $\tilde{\omega}^{\prime}$ presents a greater challenge; determining its probability density function in a closed form appears to be an intractable problem. We instead independently examine its two constitute components $\tau$ and $\nu$ (or, more precisely, their random variables $\boldsymbol{\tau}$ and $\boldsymbol{\nu})$ as delineated in (10). The form of $\boldsymbol{\tau}$ is very similar to that of $\boldsymbol{\xi}$. The difference lies in the correlation among the random variables that comprise it. The random variables $\boldsymbol{a}$ and $\boldsymbol{f}$ are correlated, as are $\boldsymbol{b}$ and $\boldsymbol{e}$. The resultant pdf of $\boldsymbol{\tau}$ (see Appendix A) is

$$
f(\tau)=\frac{\frac{|K|}{\left(\sigma_{a}^{4} \sigma_{c}^{2}\right)}}{2\left(\frac{|K|}{\left(\sigma_{a}^{4} \sigma_{c}^{2}\right)+\tau^{2}}\right)^{3 / 2}}
$$

where $K$ is given in (21). Similar to the localization term $\boldsymbol{\xi}, \boldsymbol{\tau}$ is also zero mean with infinite variance. Its absolution deviation is finite with value

$$
\mathrm{E}[|\boldsymbol{\tau}|]=\sqrt{\frac{|K|}{\left(\sigma_{a}^{4} \sigma_{c}^{2}\right)}} .
$$

The second term $\boldsymbol{\nu}$ is the product of $\boldsymbol{\xi}$ and $\boldsymbol{\chi}$, also shown in (10). The RV $\boldsymbol{\xi}$ is the localization term of $\tilde{\boldsymbol{\omega}}$ discussed in Section III-B. Equation (10) indicates that both $\boldsymbol{\xi}$ and $\boldsymbol{\chi}$ are slightly different functions of the same RVs: $\boldsymbol{a}, \boldsymbol{b}, \boldsymbol{c}$, and $\boldsymbol{d}$. To better elucidate their interrelation we convert them into polar form, setting $a=r_{1} \cos \phi_{1}, b=r_{1} \sin \phi_{1}, c=r_{2} \cos \phi_{2}$, and $\boldsymbol{d}=\boldsymbol{r}_{\mathbf{2}} \sin \boldsymbol{\phi}_{\mathbf{2}}$. The random variables $\boldsymbol{r}_{\mathbf{1}}$ and $\boldsymbol{r}_{\mathbf{2}}$ have Rayleigh distributions while $\phi_{\mathbf{1}}$ and $\phi_{\mathbf{2}}$ are distributed uniformly over the interval $[-\pi: \pi]$ [24]. Substituting these values, we find $\boldsymbol{\xi}=\boldsymbol{r}_{\mathbf{2}} / \boldsymbol{r}_{\mathbf{1}} \sin \left(\boldsymbol{\phi}_{\mathbf{2}}-\boldsymbol{\phi}_{\mathbf{1}}\right)$ and $\boldsymbol{\chi}=\boldsymbol{r}_{\mathbf{2}} / \boldsymbol{r}_{\mathbf{1}} \cos \left(\boldsymbol{\phi}_{\mathbf{2}}-\boldsymbol{\phi}_{\mathbf{1}}\right)$. We can simplify further by letting $\phi=\phi_{2}-\phi_{1}$, where $\phi$ is uniformly distributed over $[-\pi: \pi]$. The final forms of $\boldsymbol{\xi}, \boldsymbol{\chi}$, and $\boldsymbol{\nu}$ are

$$
\begin{aligned}
& \boldsymbol{\xi}=\frac{\boldsymbol{r}_{\mathbf{2}}}{\boldsymbol{r}_{\mathbf{1}}} \sin \boldsymbol{\phi} \\
& \chi=\frac{\boldsymbol{r}_{\mathbf{2}}}{\boldsymbol{r}_{\mathbf{1}}} \cos \boldsymbol{\phi} \\
& \boldsymbol{\nu}=\boldsymbol{\xi} \chi=\left(\frac{\boldsymbol{r}_{\mathbf{2}}}{\boldsymbol{r}_{\mathbf{1}}}\right)^{2} \cos \boldsymbol{\phi} \sin \boldsymbol{\phi} .
\end{aligned}
$$

The RV $\nu$ has zero mean, infinite variance, and an infinite absolute deviation (see Appendix B).

\section{Effects of Constraints}

In the previous subsections we found the distribution for the localization term $\boldsymbol{\xi}$ of $\tilde{\boldsymbol{\omega}}$ and the distributions for the components $\tau$ and $\boldsymbol{\nu}$ that comprise $\tilde{\omega}^{\prime}$. All three of these pdfs have heavy tails. In fact, all have infinite variances. The distribution for $\tilde{\omega}^{\prime}$ does not even have a finite absolute deviation. Accordingly, we would expect to find numerous regions in which $\tilde{\boldsymbol{\omega}}$ and/or $\tilde{\boldsymbol{\omega}}^{\prime}$ are too large (in magnitude) to recover meaningful disparities due to reasons discussed in Section II-B. In this subsection, we examine the ability of the constraints given in (11)-(13) to identify these regions of phase instability.

Before delving into the mathematics it may be useful to provide an intuitive explanation for the subsequent derivations. Since excessively deviant values of $\tilde{\boldsymbol{\omega}}$ and $\tilde{\boldsymbol{\omega}}^{\prime}$ result in erroneous phase measurements, we wish to determine the mitigating effects of imposing the constraints. Ultimately, we wish to discover insight into the behavior of the conditional distributions of $\tilde{\boldsymbol{\omega}}$ and $\tilde{\boldsymbol{\omega}}^{\prime}$ given that the constraints are met. As previously stated, the following derivations assume any real, symmetric windowing function $g(x)$ that results in a quadrature filter. To gain perspective it may be helpful to look ahead to Fig. 1 which plots the effects of the constraints on the constituents of $\tilde{\boldsymbol{\omega}}$ and $\tilde{\boldsymbol{\omega}}^{\prime}$ specifically for the Gaussian window (i.e., Gabor functions).

We begin the derivations by first examining the effects of the constraints on the random variables $\boldsymbol{\xi}, \boldsymbol{\tau}$, and $\boldsymbol{\nu}$. The first constraint given in (11) requires that the localization term $\boldsymbol{\xi}$ fall within a certain range. This will, in general, reduce the spread of the RV's $\boldsymbol{\xi}, \boldsymbol{\tau}$, and $\boldsymbol{\nu}$ (see Appendix C)

$$
\begin{aligned}
\mathrm{E}\left[|\xi||| \xi \mid<\rho_{1}\right]= & \frac{1}{\rho_{1}}\left(\frac{\sigma_{c}}{\sigma_{a}}\left[\frac{\sigma_{c}^{2}}{\sigma_{a}^{2}}+\rho_{1}^{2}\right]^{1 / 2}-\frac{\sigma_{c}^{2}}{\sigma_{a}^{2}}\right) \\
\mathrm{E}\left[|\tau||| \xi \mid<\rho_{1}\right]= & \frac{2}{\pi \rho_{1}}\left(\frac{|K|}{\sigma_{a}^{4} \sigma_{c}^{2}}\right)^{1 / 2}\left(\frac{\sigma_{c}^{2}}{\sigma_{a}^{2}}+\rho_{1}^{2}\right)^{1 / 2} \\
& \times \tan ^{-1}\left(\frac{\sigma_{a}}{\sigma_{c}} \rho_{1}\right) \\
\mathrm{E}\left[|\nu||| \xi \mid<\rho_{1}\right]= & \frac{1}{\pi \rho_{1}} \frac{\sigma_{c}^{2}}{\sigma_{a}^{2}}\left(\frac{\sigma_{a}^{2}}{\sigma_{c}^{2}}+\rho_{1}^{2}\right)^{1 / 2} \\
& \times \ln \left(1+\frac{\sigma_{c}^{2}}{\sigma_{a}^{2}} \rho_{1}^{2}\right) .
\end{aligned}
$$

In fact, the variances of all three RV's are now finite, whereas previously their variances were infinite as was the absolute deviation of $\nu$.

Before considering the second constraint given in (12), we will reexamine both constraints in light of the polar representations of $\boldsymbol{\xi}$ and $\boldsymbol{\chi}$ shown in (26) and (27). In polar coordinates, the constraints become

$$
\begin{aligned}
& |\xi|=\left|\frac{r_{2}}{r_{1}} \sin \phi\right|<\rho_{1} \\
& |\chi|=\left|\frac{r_{2}}{r_{1}} \cos \phi\right|<\rho_{2} .
\end{aligned}
$$

Using these constraints in the fashion suggested by (11) and (12) amounts to forming a rectangular decision region in 


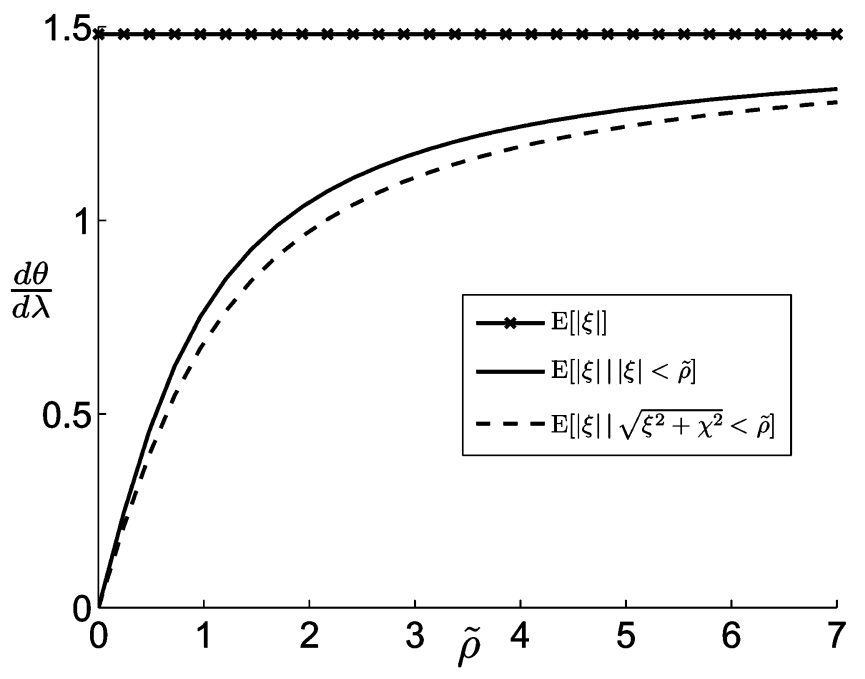

(a)

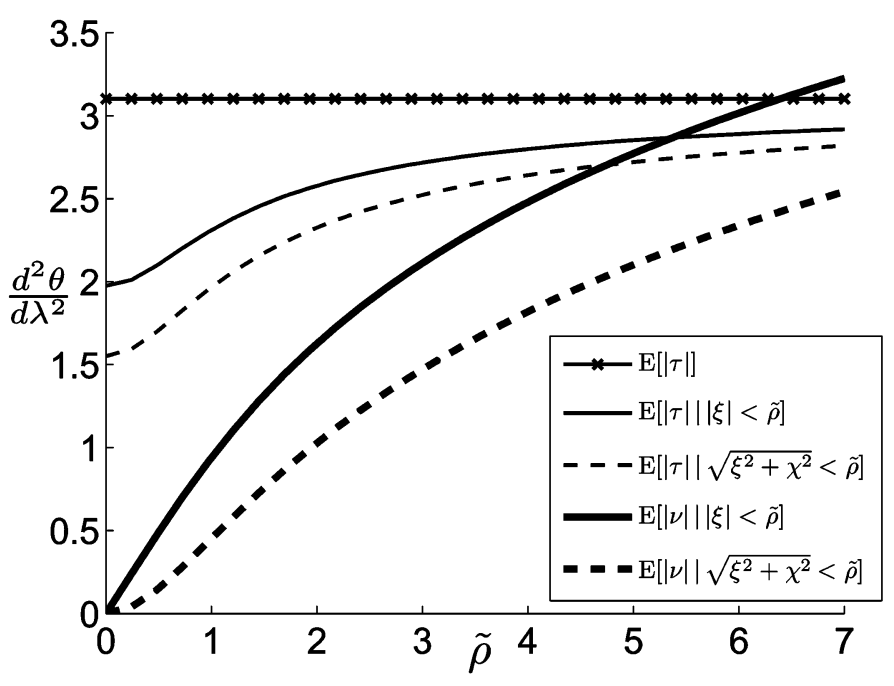

(b)

Fig. 1. $\mathrm{E}[|\boldsymbol{\xi}|], \mathrm{E}[|\boldsymbol{\tau}|]$, and $\mathrm{E}[|\boldsymbol{\nu}|]$ with respect to constraints for Gabor kernels $(\beta=1)$ responding to white noise.

$(\xi, \chi)$ space. Observing the polar forms of the constraints and remembering that $\phi$ is uniformly distributed suggests that a circular decision region like that delineated in (13) would be more appropriate

$$
\sqrt{\chi^{2}+\xi^{2}}=\sqrt{\left(\frac{r_{2}}{r_{1}}\right)^{2}\left(\cos ^{2} \phi+\sin ^{2} \phi\right)}=\frac{r_{2}}{r_{1}}<\rho_{3} .
$$

Incorporating this constraint we find that (see Appendix C)

$$
\begin{aligned}
& \mathrm{E}\left[|\boldsymbol{\xi}| \mid \sqrt{\chi^{2}+\xi^{2}}<\rho_{3}\right] \\
& =\frac{2}{\pi \rho_{3}} \frac{\sigma_{c}^{2}}{\sigma_{a}^{2}}\left(\frac{1}{\rho} \frac{\sigma_{a}}{\sigma_{c}} \tan ^{-1}\left[\frac{\sigma_{a}}{\sigma_{c}} \rho_{3}\right]\left[\frac{\sigma_{c}^{2}}{\sigma_{a}^{2}}+\rho_{3}^{2}\right]-1\right) \\
& \mathrm{E}\left[\mid \boldsymbol{\tau} \| \sqrt{\chi^{2}+\xi^{2}}<\rho_{3}\right] \\
& =\frac{1}{\rho_{3}^{2}} \frac{\sigma_{c}}{\sigma_{a}}\left[\frac{|K|}{\sigma_{a}^{4} \sigma_{c}^{2}}\left(\frac{\sigma_{c}^{2}}{\sigma_{a}^{2}}+\rho_{3}^{2}\right)\left(\frac{\sigma_{a}}{\sigma_{b}}\left[\frac{\sigma_{c}^{2}}{\sigma_{a}^{2}}+\rho_{3}^{2}\right]-1\right)\right]^{1 / 2}
\end{aligned}
$$$$
\begin{aligned}
& \mathrm{E}\left[|\boldsymbol{\nu}| \mid \sqrt{\chi^{2}+\xi^{2}}<\rho_{3}\right] \\
& \quad=\frac{1}{\pi} \frac{\sigma_{c}^{2}}{\sigma_{a}^{2}}\left(\frac{\frac{\sigma_{c}^{2}}{\sigma_{a}^{2}}+\rho_{3}^{2}}{\rho_{3}^{2}}\left[\ln \left(\frac{\frac{\sigma_{c}^{2}}{\sigma_{a}^{2}}+\rho_{3}^{2}}{\frac{\sigma_{c}^{2}}{\sigma_{a}^{2}}}\right)\right]-1\right) .
\end{aligned}
$$

Using the triangle inequality, we can now establish a bound on the expected nonlinearity $\mathrm{E}\left[\left|\tilde{\boldsymbol{\omega}}^{\prime}\right|\right]$ in regions satisfying (13)

$$
\begin{aligned}
& \mathrm{E}\left[\left|\tilde{\boldsymbol{\omega}}^{\prime}\right| \mid \sqrt{\chi^{2}+\xi^{2}}<\rho_{3}\right] \\
& \quad=\mathrm{E}\left[|\boldsymbol{\tau}-2 \boldsymbol{\nu}| \mid \sqrt{\chi^{2}+\xi^{2}}<\rho_{3}\right] \\
& \quad \leq \mathrm{E}\left[|\boldsymbol{\tau}| \mid \sqrt{\chi^{2}+\xi^{2}}<\rho_{3}\right] \\
& +2 \mathrm{E}\left[|\boldsymbol{\nu}| \mid \sqrt{\chi^{2}+\xi^{2}}<\rho_{3}\right] .
\end{aligned}
$$

We have now demonstrated that the constraint in (13) serves to limit the second derivative of phase $\tilde{\omega}^{\prime}$. In fact, the second term $\nu$ can be completely controlled by this constraint. Unfortunately, though the first term $\tau$ also tends to be reduced, it can still obtain arbitrarily large values. To further restrict this term, and consequently $\tilde{\omega}^{\prime}$, we introduce the additional constraint

$$
|\tau|<\rho_{4}
$$

The rationale and appropriateness of this condition will become more apparent in later sections when actual kernels are evaluated.

\section{GABOR FUNCTIONS}

In this section, we use the previous results to analyze a specific kernel, the Gabor function. For a Gabor kernel $h(x)$, the windowing function $g(x)$ in (7) is the Gaussian. The Gaussian is often the window of choice because of its joint optimality with respect to support in both time and space [25]. In addition to stereo registration [9], [26], [27], the Gabor function has found many useful applications in areas such as texture analysis [28], [29]. Furthermore, the Gabor function is a common model of the simple receptive fields in the visual cortex [30]-[32]. Normalizing $h(x)$ to have unit energy yields

$$
h(x)=\left[\sqrt{\pi} \sigma_{g}\right]^{-1 / 2} \exp \left(-\frac{x^{2}}{2 \sigma_{g}^{2}}\right) \exp \left(j \omega_{o} x\right) .
$$

The Fourier transform of the Gabor kernel is a Gaussian shifted by the modulation frequency $\omega_{o}$ with standard deviation $\sigma_{\omega}=$ $1 / \sigma_{g}$. In order for a Gabor function to adequately approximate a quadrature filter, $\omega_{o}$ must be much greater than $\sigma_{\omega}$. To ensure this relationship across different frequencies Gabor functions of constant relative bandwidth $\beta$ are usually assumed, i.e., $\beta=$ $\log _{2}\left[\left(\omega_{o}+\sigma_{\omega}\right) /\left(\omega_{o}-\sigma_{\omega}\right)\right]$. A bandwidth less than or equal to one octave $(\beta \leq 1)$ is usually sufficient to well approximate a quadrature pair filter. With $\beta=1$ the Gaussian envelope in the frequency domain crosses DC at three standard deviations. A perfect quadrature pair filter requires a zero response at DC. For a more complete discussion on the selection of parameters for Gabor functions, see [33]. 
Incorporating $h(x)$ into (7), the covariance matrix in (21) becomes

$$
K=\left[\begin{array}{ccc}
\frac{1}{2} & 0 & -\frac{1}{4 \sigma_{g}^{2}} \\
0 & \frac{1}{4 \sigma_{g}^{2}} & 0 \\
-\frac{1}{4 \sigma_{g}^{2}} & 0 & \frac{3}{8 \sigma_{g}^{4}}
\end{array}\right] .
$$

Using these values, we can now illustrate the effects of the constraints on the first and second derivatives of phase as formulated in (23), (25), (29)-(31), and (35)-(37). Fig. 1 plots these equations for Gabor kernels with relative bandwidth $\beta=1$. The $X$ axes indicate the value of the normalized constraint $\tilde{\rho}_{i}$, where $\rho_{i} / \sigma_{\omega}=\tilde{\rho}_{i}$. Since the $Y$ axes are expressed in terms of wavelength $\lambda_{o}$, all Gabor kernels with the same relative bandwidth produce identical curves. This is a consequence of the following relation: $d \theta / d \lambda=d \theta / d x d x / d \lambda=d \theta / d x \lambda_{o}$, where $\lambda_{o}=2 \pi / \omega_{o}$.

We next test the ability of the constraints in (11)-(13) and (39) to identifying regions where phase-based disparity measurements are poor. The constraints can be considered features; and they can be measured at every point we calculate disparity. These features (or some functions of them) can be used to make a decision as to whether we can trust the disparity estimate at that point. The points can be separated into two sets: points at which (3) produces correct disparity estimates (set $\mathcal{C}_{1}$ ) and points at which (3) produces incorrect disparity estimates (set $\mathcal{C}_{2}$ ). Specifically, set $\mathcal{C}_{1}$ contains those points with a calculated disparity estimate within $25 \%$ of the true disparity. Set $\mathcal{C}_{2}$ contains all points not in $\mathcal{C}_{1}$. The goal is to use a set of features that will allow us to accept the greatest number of points in $\mathcal{C}_{1}$ while rejecting the greatest number of points in $\mathcal{C}_{2}$.

Prior to evaluating the performance of different sets of features, let us consider the candidate features themselves. In order to detect regions of phase nonlinearity, the literature advocates using the features $|\xi|$ and $|\chi|$ by thresholding them above a certain value. This forms a rectangular decision region in $(\xi, \chi)$ space. In Section III-D, we suggested that a circular decision region was more appropriate. Such a region is constructed by thresholding the 1-D feature $\sqrt{\xi^{2}+\chi^{2}}$. Furthermore, in Section II-B, we suggested that the second derivative of phase $\tilde{\omega}^{\prime}$ was a better indicator of phase nonlinearity. The variables $\xi$ and $\chi$ represent two of the three components of $\tilde{\omega}^{\prime}(10)$. The use of the final component $\tau$ is absent from the literature. If the second derivative of phase is valuable, its addition should improve classification.

It may appear that the best feature simply would be the second derivative itself. This is not true. Consider the case where the first derivative is very large, but the second derivative is zero. Though phase may be linear, the constant of linearity is so large that we will incur errors due to phase wrapping. Consequently, at least one feature must restrict the size of $\xi$. The second derivative is not guaranteed to do this. The previously discussed features $|\xi|$ and $\sqrt{\xi^{2}+\chi^{2}}$ both implement this restriction.

The next question is how to incorporate the second derivative as a feature. Again, the simple solution would be to use the features $|\xi|$ and $\left|\tilde{\omega}^{\prime}\right|$. While this combination may work very well in theory, in practice it poses complications. Since $\xi$ is a component of $\tilde{\omega}^{\prime}$, the features are highly dependent. This results in an optimal decision region that is irregularly shaped, and consequently, not well approximated by applying a separate threshold to each feature (as we said previously, this forms rectangular decision regions). When thresholding it is important that the features be as independent as possible. For this reason, we propose incorporating the second derivative by using the following features: $\sqrt{\xi^{2}+\chi^{2}}$ and $|\tau|$. Fig. 2 compares the use of three different feature sets. The first feature set uses the methodology most often advocated in the literature: $|\xi|$ and $|\chi|$. The second set uses $\sqrt{\xi^{2}+\chi^{2}}$, altering the decision region from a rectangle to a circle. The final set uses both $\sqrt{\xi^{2}+\chi^{2}}$ and $|\tau|$. Assuming that each feature is thresholded at a certain value, Fig. 2(a) compares the resulting receiver operator characteristic curves for each set of features when applied to white noise with a constant disparity of $\lambda_{o} / 8$. Approximately $96 \%$ of the points belong to set $\mathcal{C}_{1}$. Interestingly, the circular decision region $\sqrt{\xi^{2}+\chi^{2}}$ slightly outperforms the rectangular region delineated by $|\xi|$ and $|\chi|$. This is remarkable because the space of possible decision regions for $|\xi|$ and $|\chi|$ has two degrees of freedom (length and width), while the circular decision region for $\sqrt{\xi^{2}+\chi^{2}}$ has only one (radius). A fairer comparison might be to allow arbitrary elliptical regions.

Since the results in this paper were derived under the assumption of a white noise signal, the question of their extensibility remains. To address this concern we recreate Fig. 2(a), this time replacing the white noise image with concatenated scan lines of a natural scene. Obviously, these concatenated scan lines do not replicate the prospective transformations, occlusions, and other higher order effects that exist between two stereo images, but they do better represent the frequencies found in real images. Fig. 2(b) demonstrates that, though the overall performance of each set is slightly worse than with white noise, the order of efficacy remains the same. Additionally, the performance degradation for the feature set containing $\sqrt{\xi^{2}+\chi^{2}}$ and $|\tau|$ is significantly less than that of the other feature sets.

At this point, it is useful to note that Gabor filters are only approximations of true quadrature filters. In reality, the real component has some DC bias that would not be present if the real and imaginary components were actually Hilbert transforms of one another. For zero mean images, such as the one considered in Fig. 2(a), such a bias has no effect. Real images, such as the one used to create Fig. 2(b), possess a frequency spectrum better modeled by the function $1 / f$ [34]. The relatively large DC component in such images can disrupt phase measurements. To mitigate these disruptive effects the DC sensitivity of the Gabor filter can be removed. Fig. 2(c) illustrates the attendant increase in performance for the natural scene when the DC component is eliminated. The thin dashed lines are the previous plots from Fig. 2(b) and are included for reference.

Another question about the extensibility of our results arises as we move away from the point of expansion $\delta=0$ of the Taylor series of $\Delta \theta$. This expansion, shown in (2), considers both the first-order $\tilde{\omega}$ and the second-order $\tilde{\omega}^{\prime}$ terms. Due to the effect of higher order terms, the accuracy should degrade as $\delta$ moves away from zero. It is important to investigate whether or not this degradation is significant. That 


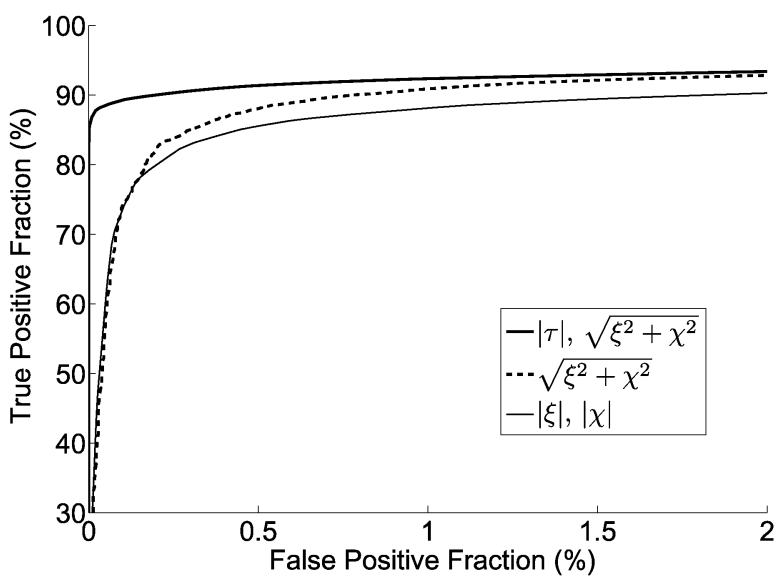

(a)

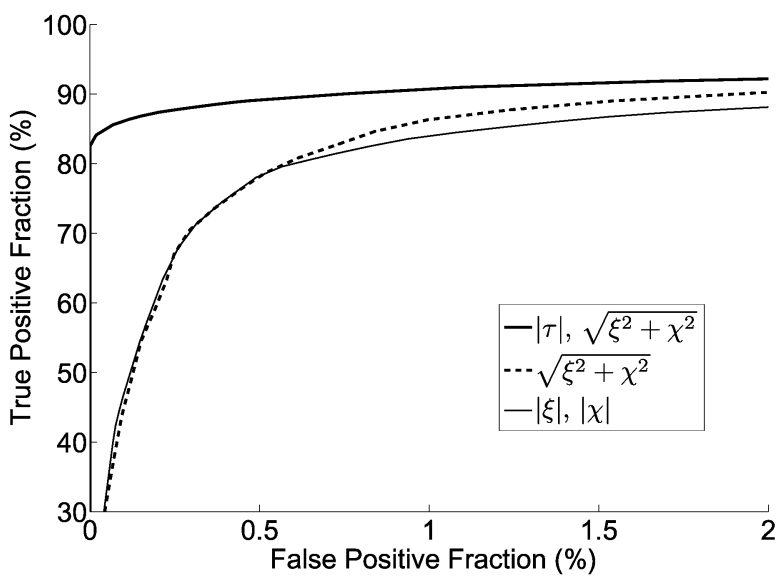

(b)

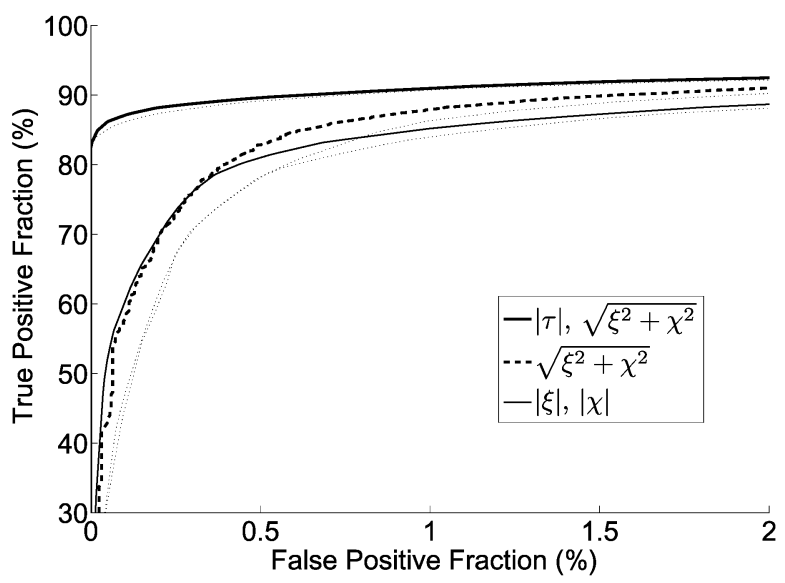

(c)

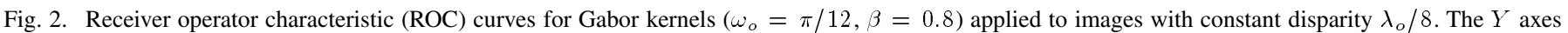

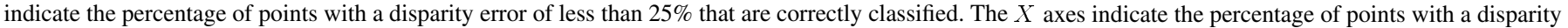

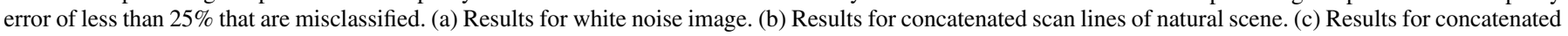
scan lines of natural scene with DC component removed from Gabor filter. Thin dashed lines are the plots from (b) included for reference.

is, in regions satisfying the constraint given by (13) will the model reasonably predict the actual phase difference $\Delta \theta$ ? To evaluate this we consider two quantities. The first quantity $\mathrm{E}\left[\boldsymbol{\Delta} \boldsymbol{\theta}(\delta) \mid \sqrt{\xi^{2}+\chi^{2}}<\rho_{3}\right]$ measures the mean phase difference $\Delta \theta$ as a function of the displacement $\delta$. The second $\mathrm{E}\left[|\boldsymbol{\Delta} \boldsymbol{\theta}(\delta)-\mathrm{E}[\boldsymbol{\Delta} \boldsymbol{\theta}(\delta)]| \mid \sqrt{\xi^{2}+\chi^{2}}<\rho_{3}\right]$ evaluates the deviation about the mean as a function of $\delta$. For convenience, we will henceforth refer to these quantities as $E[\Delta \boldsymbol{\theta}]$ and $\mathrm{E}[\mid \boldsymbol{\Delta} \boldsymbol{\theta}-\mathrm{E}[\boldsymbol{\Delta} \boldsymbol{\theta}]]]$, leaving implicit the variable $\delta$ and the dependence on $\sqrt{\xi^{2}+\chi^{2}}<\rho_{3}$.

Combining the second-order Taylor expansion in (2) with the results from Section III-D, these expected values can be expressed as

$$
\begin{aligned}
\mathrm{E}[\boldsymbol{\Delta} \boldsymbol{\theta}] & \approx \mathrm{E}\left[\omega_{o}+\boldsymbol{\xi}\right] \delta+\frac{1}{2} \mathrm{E}[\boldsymbol{\tau}+2 \boldsymbol{\nu}] \delta^{2}=\omega_{o} \delta \\
\mathrm{E}[|\boldsymbol{\Delta} \boldsymbol{\theta}-\mathrm{E}[\boldsymbol{\Delta} \boldsymbol{\theta}]|] & \approx \mathrm{E}\left[\left|\left(\omega_{o}+\boldsymbol{\xi}\right) \delta+\frac{1}{2}(\boldsymbol{\tau}+2 \boldsymbol{\nu}) \delta^{2}-\omega_{o} \delta\right|\right] \\
& \approx \mathrm{E}[|\boldsymbol{\xi}|] \delta+\frac{1}{2}(\mathrm{E}[|\boldsymbol{\tau}|]+2 \mathrm{E}[|\boldsymbol{\nu}|]) \delta^{2} .
\end{aligned}
$$

Equation (42) results from the zero-mean properties of $\boldsymbol{\xi}, \boldsymbol{\tau}$, and $\boldsymbol{\nu}$. Obtaining (43) requires incorporating the inequality in (38).
In [18], Fleet also presents a model for both these quantities

$$
\begin{aligned}
\mathrm{E}[\boldsymbol{\Delta} \boldsymbol{\theta}] & =\omega_{o} \delta \\
\mathrm{E}[|\boldsymbol{\Delta} \boldsymbol{\theta}-\mathrm{E}[\boldsymbol{\Delta} \boldsymbol{\theta}]|] & =\sqrt{\exp \left(\frac{-\delta^{2}}{2 \sigma_{g}^{2}-1}\right) .}
\end{aligned}
$$

Fig. 3 plots these models as a function of the disparity for both a white noise image and concatenated scan lines of a natural scene. The disparity is not given explicitly in terms of $\delta$ but instead as a fraction of the wavelength. Additionally, Fig. 3 provides the simulated values for $\mathrm{E}[\boldsymbol{\Delta} \boldsymbol{\theta}]$ and $\mathrm{E}[|\boldsymbol{\Delta} \boldsymbol{\theta}-\mathrm{E}[\boldsymbol{\Delta} \boldsymbol{\theta}]|]$ determined explicitly from the data. The simulation and the models assume that measurements are made in regions of linear phase, i.e., points satisfying $\sqrt{\xi^{2}+\chi^{2}}<\rho_{3}$, where $\rho_{3}=1$. The threshold $\rho_{3}=1$ is commonly advocated in the literature [10]. As we can see from the figure, both models adequately predict the simulated results for both the white noise and natural scene images. Finally, with the goal of visually demonstrating the advantages of (39), we present a random-dot stereo pair whose disparity is the isotropic Gaussian shown in Fig. 4(a). With a Gabor kernel $\left(\beta=1, \omega_{0}=\pi / 6\right)$, disparity is estimated using (3), producing the images in Fig. 4(b) and (c). Regions in Fig. 4(b) violating the criteria $\sqrt{\xi^{2}+\chi^{2}}<1.27$ were removed. 


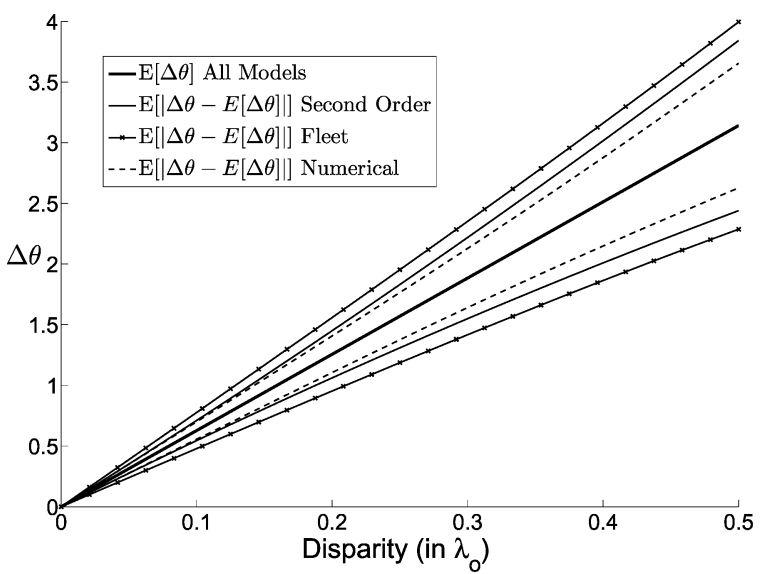

(a)

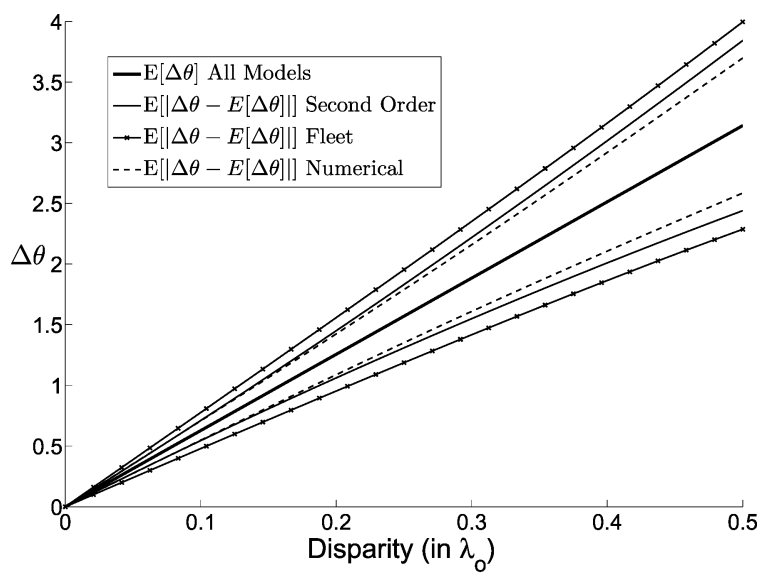

(b)

Fig. 3. Comparison of mean phase difference $\mathrm{E}[\boldsymbol{\Delta} \boldsymbol{\theta}]$ and expected deviation of phase difference about mean $\mathrm{E}[|\boldsymbol{\Delta} \boldsymbol{\theta}-\mathrm{E}[\boldsymbol{\Delta} \boldsymbol{\theta}]|]$ between numerically simulated results, our second-order model [(42) and (43), and model proposed by Fleet ((44) and (45)]. The phase difference is measured at points satisfying $\sqrt{\xi^{2}+\chi^{2}}<1$. (a) Results for white noise image. (b) Results for concatenated scan lines of natural scene.

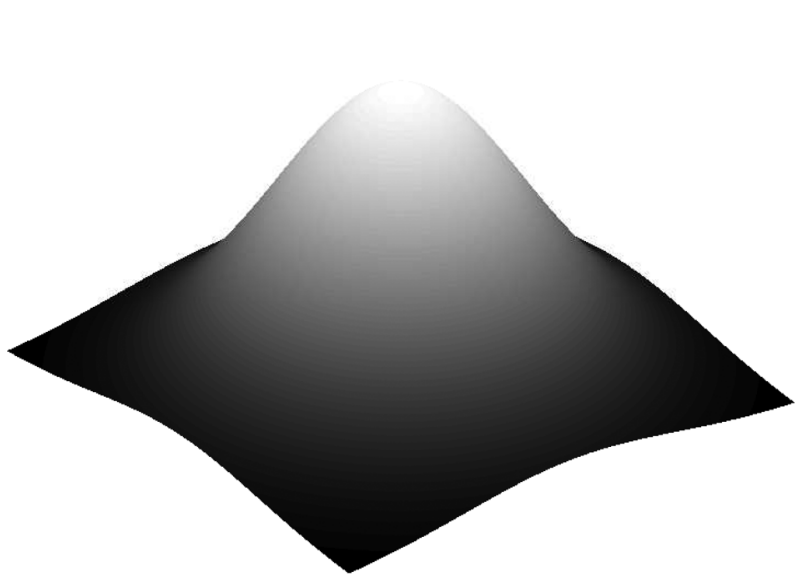

(a)

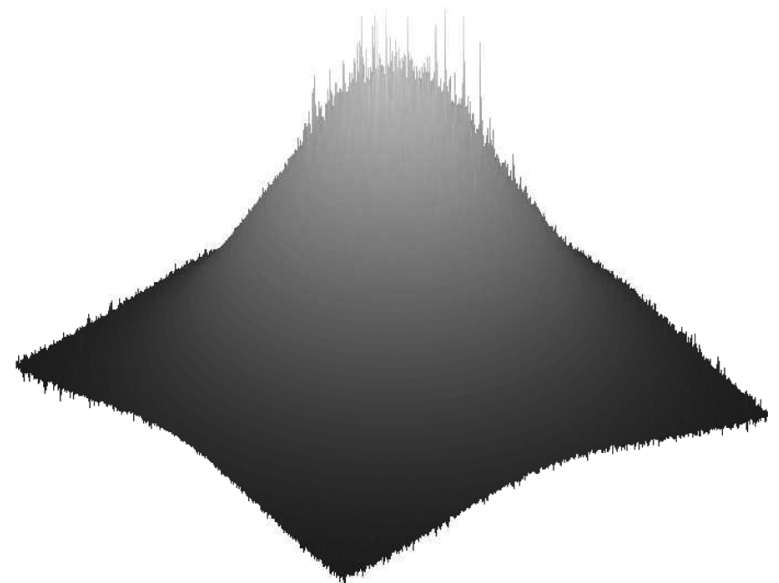

(b)

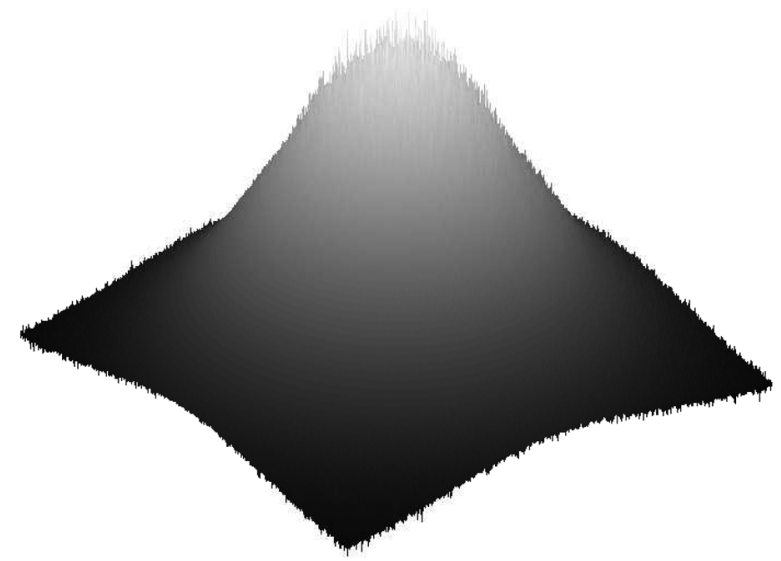

(c)

Fig. 4. (a) Ideal disparity map. (b) Disparity map using $\sqrt{\xi^{2}+\chi^{2}}<1.27$. (c) Disparity map using $\sqrt{\xi^{2}+\chi^{2}}<1.45$ and $|\tau|<1.34$.

Regions in Fig. 4(c) violating $\sqrt{\xi^{2}+\chi^{2}}<1.45$ or $|\tau|<1.34$ were removed. Eliminated points were replaced by linear interpolation. To ensure a fair comparison, both sets of constraints were chosen so as to remove the same number of measurements $(24 \%)$. The valid points remaining in both disparity maps are 95\% identical. Still, the images shown in Fig. 4(b) and (c) are visually different. This difference manifests as several isolated spikes in Fig. 4(b), which are both less frequent and less prominent in Fig. 4(c). The largest spikes occur at the peak of the Gaussian where the disparity is the greatest. This is understandable since the greater the disparity, the greater the error resulting from nonlinearities. 
TABLE I

ERROR METRICS FOR DISPARITY MAPS IN FIG. 4. (A) MSE. (B) SSIM

(a)

\begin{tabular}{|c|c|c|c|}
\hline Top N\% & Fig. 4(b) MSE & Fig. 4(c) MSE & Gain(\%) \\
\hline \hline 0.1 & 0.4560 & 0.3708 & 22.9896 \\
\hline 1 & 0.2331 & 0.2101 & 10.9518 \\
\hline 2 & 0.1861 & 0.1710 & 8.8463 \\
\hline 5 & 0.1357 & 0.1276 & 6.3401 \\
\hline 10 & 0.1053 & 0.1005 & 4.7955 \\
\hline 15 & 0.0901 & 0.0865 & 4.1061 \\
\hline 100 & 0.0383 & 0.0372 & 2.9586 \\
\hline
\end{tabular}

(b)

\begin{tabular}{|c|c|c|c|}
\hline Top N\% & Fig. 4(b) SSIM & Fig. 4(c) SSIM & Gain(\%) \\
\hline \hline 0.1 & 0.9481 & 0.9725 & 2.5823 \\
\hline 1 & 0.9799 & 0.9847 & 0.4869 \\
\hline 2 & 0.9853 & 0.9881 & 0.2792 \\
\hline 5 & 0.9907 & 0.9920 & 0.1281 \\
\hline 10 & 0.9937 & 0.9944 & 0.0673 \\
\hline 15 & 0.9951 & 0.9955 & 0.0441 \\
\hline 100 & 0.9988 & 0.9988 & 0.0048 \\
\hline
\end{tabular}

Table I(a) provides the mean squared error (MSE) between the true disparity map shown in Fig. 4(a) and the estimates shown in Fig. 4(b) and (c). Since the majority of the disparity estimates are identical, it is instructive to calculate the MSE only considering the $N \%$ of the points with the largest squared error. These MSE calculations, evaluated for several values of $N \%$, are provided in the table. To further confirm these results we replace MSE with the structural similarity index metric (SSIM) developed by Wang and Bovik [35], [36]. SSIM offers an alternative to MSE for assessing image quality. Based on properties of the human visual system the SSIM metric provides a quality index by combining three metrics based on the following: loss of correlation, mean distortion, and variance distortion. The final value ranges from $[-1,1]$, with 1 signifying no error. Using qualitative experiments, the SSIM metric has been shown to better model a human's perceived quality measure. Substituting SSIM for MSE, Table I(b) reprises Table I(a).

\section{CONCLUSION}

In this paper, we were able to derive probability density functions for the components of both the first and second derivatives of localized phase. We quantified the effects on these pdfs caused by constraining their domains to regions of linear phase. In order to identify these regions, we proposed using the second derivative of phase. We then described how prevalent methods proposed in the literature can be seen as approximations of the second derivative. We empirically validated these assertions and their extensibility by comparing the different methods on white noise, a natural scene, and a random-dot stereo pair.

Undoubtedly, local phase is a powerful means for recovering the disparity relating two different signals. Unfortunately, we lack a rigorous mathematical and intuitive framework for truly understanding it. At times, however, there are works such as [18] that expand our knowledge base and improve our insight into its machinations. In this paper we have reexamined many of the current perceptions and paradigms associated with phase nonlinearity and have reformulated them in terms of something with which we are very familiar: the second derivative. Additionally, we have verified this interpretation by demonstrating its efficacy in detecting regions in which phase measurements break down.

\section{APPENDIX A \\ Probability DENSITY FunCtion DERIVATIONS}

Consider the zero mean Gaussian random variables $\boldsymbol{a}, \boldsymbol{b}, \boldsymbol{c}, \boldsymbol{d}$, $\boldsymbol{e}$, and $\boldsymbol{f}$. Let $K$ defined in (21) be the covariance matrix relating $\boldsymbol{a}, \boldsymbol{c}$, and $\boldsymbol{e}$ and also relating $\boldsymbol{b}, \boldsymbol{d}$, and $\boldsymbol{f}$. These matrices represent the only dependencies between the six random variables (i.e., $\boldsymbol{a}$, $\boldsymbol{c}$, and $\boldsymbol{e}$ are independent with respect to $\boldsymbol{b}, \boldsymbol{d}$, and $\boldsymbol{f}$ ). Their joint probability density function (pdf) is

$$
\begin{aligned}
& f(a, b, c, d, e, f) \\
& =\frac{1}{(2 \pi)^{3}|K|}
\end{aligned}
$$

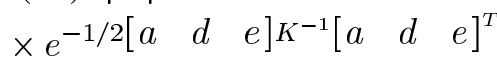

$$
\begin{aligned}
& \left.\times e^{-1 / 2[b} \quad c \quad f\right] K^{-1}\left[\begin{array}{lll}
b & c & f
\end{array}\right]^{T}
\end{aligned}
$$

where $|K|$ is the determinant of $K$. In Sections VII-A and B, we will consider the properties of functional combinations of these random variables.

At this point, it is important to note that Fleet [23] derived a distribution for $\boldsymbol{\xi}$ similar to that which immediately follows. His derivations considered the more general case where $\boldsymbol{a}, \boldsymbol{b}, \boldsymbol{c}$, and $\boldsymbol{d}$ were not all independent. He did not, however, consider the additional components $\boldsymbol{e}$ and $\boldsymbol{f}$.

\section{A. Probability Density Functions for $\boldsymbol{\xi}$ and $\boldsymbol{\tau}$}

The goal of this section is to find the joint and marginal pdfs of the random variables $\boldsymbol{\xi}$ and $\boldsymbol{\tau}$, where $\boldsymbol{\xi}=(\boldsymbol{a} \boldsymbol{d}-\boldsymbol{b} \boldsymbol{c}) /\left(\boldsymbol{a}^{2}+\boldsymbol{b}^{2}\right)$ and $\boldsymbol{\tau}=(\boldsymbol{a} \boldsymbol{f}-\boldsymbol{b} \boldsymbol{e}) /\left(\boldsymbol{a}^{2}+\boldsymbol{b}^{2}\right)$. Consider the following functions of the random variables $\boldsymbol{a}, \boldsymbol{b}, \boldsymbol{c}, \boldsymbol{d}, \boldsymbol{e}$, and $\boldsymbol{f}: \boldsymbol{w}=\boldsymbol{a d}-\boldsymbol{b c}$, $\boldsymbol{x}=\boldsymbol{a}^{2}+\boldsymbol{b}^{2}, \boldsymbol{y}=\boldsymbol{a}^{2}, \boldsymbol{z}=\boldsymbol{b} \boldsymbol{c}, \boldsymbol{r}=\boldsymbol{a} \boldsymbol{f}-\boldsymbol{b e}$, and $\boldsymbol{s}=\boldsymbol{b e}$. The solutions of this system of equations are $a= \pm \sqrt{y}, b= \pm \sqrt{x-y}$, $c=x / \pm \sqrt{x-y}, d=(w+z) / \pm \sqrt{y}, e=s / \pm \sqrt{x-y}$ and $f=(r+s) / \pm \sqrt{y}$. Because of the \pm nature of the solutions $\pm \sqrt{y}$ and $\pm \sqrt{x-y}$, there are four unique roots (combination of solutions). The Jacobian of the these transformations is $J(w, x, y, z, r, s)=4[y(x-y)]^{3 / 2}$. Incorporating these results into (46) and simplifying, the joint pdf can be expressed as follows (for simplicity let $K^{-1}=\left[\begin{array}{ccc}2 \alpha & 0 & \gamma \\ 0 & 2 \beta & 0 \\ \gamma & 0 & 2 \delta\end{array}\right]$ )

$$
\begin{aligned}
f(w, x, y, z, r, s)= & \frac{[y(x-y)]^{-3 / 2}}{(2 \pi)^{3}|K|} \\
& \times e^{-\alpha x-\beta\left[z+\mu_{z}\right]^{2} /(x-y) y-\delta x\left[s+\mu_{s}\right]^{2} /(x-y) y} \\
& \times e^{--\gamma^{2} x^{2}+4 \delta^{2} r^{2}+4 \beta \delta w^{2} / 4 \delta x}
\end{aligned}
$$

where

$$
\mu_{z}=w(x-y) / x
$$

and

$$
\mu_{s}=2 \delta(x-y) r+\gamma x \sqrt{y(x-y)} / 2 \delta x
$$

We next integrate out the variables $z, s$, and $y$

$$
f(w, x, r)=\frac{1}{8 \pi|K| x \sqrt{\beta \delta}} e^{-\alpha x} e^{-1 / 4 \delta x\left[-\gamma^{2} x^{2}+4 \delta^{2} r^{2}+4 \beta \delta w^{2}\right]} .
$$


Continuing, consider three new functions of the random variables $\boldsymbol{x}, \boldsymbol{w}$, and $\boldsymbol{r}: \boldsymbol{\xi}=\boldsymbol{w} / \boldsymbol{x}, \boldsymbol{\lambda}=\boldsymbol{x}$, and $\boldsymbol{\tau}=\boldsymbol{r} / \boldsymbol{x}$ [since the mathematics may obfuscate matters, we reiterate that these representations of $\boldsymbol{\xi}$ and $\boldsymbol{\tau}$ correspond to the original definitions: $\boldsymbol{\xi}=(\boldsymbol{a d}-\boldsymbol{b c}) /\left(\boldsymbol{a}^{2}+\boldsymbol{b}^{2}\right)$ and $\left.\boldsymbol{\tau}=(\boldsymbol{a} \boldsymbol{f}-\boldsymbol{b e}) /\left(\boldsymbol{a}^{2}+\boldsymbol{b}^{2}\right)\right]$. The Jacobian is $J(\xi, \lambda, \tau)=1 / \lambda^{2}$. Finally, we can substitute the solutions $w=\xi \lambda, x=\lambda$, and $r=\tau \lambda$ into the previous equation for $f(w, x, r)$ and integrate with respect to $\lambda$ to arrive at the joint pdf of $\boldsymbol{\xi}$ and $\boldsymbol{\tau}$

$$
\begin{aligned}
f(\xi, \tau) & =\frac{1}{8 \pi|K| \sqrt{\beta \delta}} \int_{0}^{\infty} \lambda e^{-\lambda / 4 \delta\left[4 \delta \alpha-\gamma^{2}+4 \delta^{2} \tau^{2}+4 \beta \delta \xi^{2}\right]} d \lambda \\
& =\frac{|K|^{3 / 2}}{\pi \sigma_{a}^{5} \sigma_{c}^{4}}\left[\frac{|K|}{\sigma_{a}^{4} \sigma_{c}^{2}}\left(\frac{\sigma_{c}^{2}}{\sigma_{a}^{2}}+\xi^{2}\right)+\tau^{2}\right]^{-2}
\end{aligned}
$$

Integrating (47) with respect to $\tau$ and $\xi$ produces the distributions $f(\xi)$ and $f(\tau)$, respectively

$$
\begin{aligned}
& f(\xi)=\frac{\frac{\sigma_{c}^{2}}{\sigma_{a}^{2}}}{2\left(\frac{\sigma_{c}^{2}}{\sigma_{a}^{2}}+\xi^{2}\right)^{3 / 2}} \\
& f(\tau)=\frac{\frac{|K|}{\sigma_{a}^{4} \sigma_{c}^{2}}}{2\left(\frac{|K|}{\sigma_{a}^{4} \sigma_{c}^{2}}+\tau^{2}\right)^{3 / 2}} .
\end{aligned}
$$

Some important moments of $\boldsymbol{\xi}$ and $\boldsymbol{\tau}$ are $\mathrm{E}[\xi]=0$, $\mathrm{E}[\boldsymbol{\tau}]=0, \mathrm{E}\left[\boldsymbol{\xi}^{2}\right] \rightarrow \infty$, and $\mathrm{E}\left[\boldsymbol{\tau}^{2}\right] \rightarrow \infty$. Noting that $\int_{-\infty}^{\infty}|t| t_{o} / 2\left(t_{o}+t^{2}\right)^{3 / 2} d t=\sqrt{t_{o}}$, we see

$$
\begin{aligned}
& \mathrm{E}[|\boldsymbol{\xi}|]=\sqrt{\frac{\sigma_{c}^{2}}{\sigma_{a}^{2}}} \\
& \mathrm{E}[|\tau|]=\sqrt{\frac{|K|}{\sigma_{a}^{4} \sigma_{c}^{2}}} .
\end{aligned}
$$

It is worth noting that the pdfs given in (48) and (49) are instances of generalized Cauchy distributions [37], [38].

\section{B. Alternative Representation for $\tau$}

In the representation of $\tau$ shown in (10), the random variables $\boldsymbol{a}$ and $\boldsymbol{e}$ are dependent, as are $\boldsymbol{b}$ and $\boldsymbol{f}$. These dependencies make further derivations involving $\boldsymbol{\tau}$ more difficult. In this section, we introduce an alternative form in which these dependencies are not present. Begin by letting $\boldsymbol{s}=\alpha_{1} \boldsymbol{a}+\boldsymbol{e}$ and $\boldsymbol{t}=\alpha_{2} \boldsymbol{b}+\boldsymbol{f}$. We desire values for $\alpha_{1}$ and $\alpha_{2}$ such that $\boldsymbol{a}$ is independent of $\boldsymbol{s}$ and $\boldsymbol{b}$ is independent of $\boldsymbol{t}$. This requires $\mathrm{E}[\boldsymbol{a s}]=\mathrm{E}\left[\boldsymbol{a}\left(\alpha_{1} \boldsymbol{a}+\boldsymbol{e}\right)\right]=$ $\alpha_{1} \mathrm{E}\left[\boldsymbol{a}^{2}\right]+\mathrm{E}[\boldsymbol{a e}]=0$ and $\mathrm{E}[\boldsymbol{b} \boldsymbol{t}]=\alpha_{2} \mathrm{E}\left[\boldsymbol{b}^{2}\right]+\mathrm{E}[\boldsymbol{b} \boldsymbol{f}]=0$, i.e.,

$$
\begin{aligned}
& \alpha_{1}=-\frac{\mathrm{E}[\boldsymbol{a} e]}{\boldsymbol{a}^{2}}=\frac{\sigma_{c}^{2}}{\sigma_{a}^{2}} \\
& \alpha_{2}=-\frac{\mathrm{E}[\boldsymbol{b} \boldsymbol{f}]}{\boldsymbol{b}^{2}}=\frac{\sigma_{c}^{2}}{\sigma_{a}^{2}} .
\end{aligned}
$$

Using these values for $\alpha_{1}$ and $\alpha_{2}$, the variances of $\mathbf{s}$ and $\boldsymbol{t}$ become

$$
\sigma_{s}^{2}=\mathrm{E}\left[\left(\frac{\sigma_{c}^{2}}{\sigma_{a}^{2}} \boldsymbol{s}+\boldsymbol{e}\right)^{2}\right]=\frac{|K|}{\sigma_{a}^{4} \sigma_{c}^{2}}=\sigma_{t}^{2} .
$$

Furthermore, the random variables $\boldsymbol{a}, \boldsymbol{b}, \boldsymbol{c}, \boldsymbol{d}, \boldsymbol{s}$, and $\boldsymbol{t}$ are all uncorrelated and, consequently, independent. (refer to Section III-A if this is not clear).

Continuing, we can now express $\tau$ as follows:

$$
\boldsymbol{\tau}=\frac{\boldsymbol{a} \boldsymbol{f}-\boldsymbol{b} \boldsymbol{e}}{\boldsymbol{a}^{2}+\boldsymbol{b}^{2}}=\frac{\boldsymbol{a}\left(\alpha_{2} \boldsymbol{b}+\boldsymbol{t}\right)-\boldsymbol{b}\left(\alpha_{1} \boldsymbol{a}+\boldsymbol{s}\right)}{\boldsymbol{a}^{2}+\boldsymbol{b}^{2}}=\frac{\boldsymbol{a} \boldsymbol{t}-\boldsymbol{b} \boldsymbol{s}}{\boldsymbol{a}^{2}+\boldsymbol{b}^{2}}
$$

Because of the independence of $\boldsymbol{a}, \boldsymbol{b}, \boldsymbol{s}$, and $\boldsymbol{t}$, this form of $\boldsymbol{\tau}$ behaves similarly to $\boldsymbol{\xi}$ (where $\boldsymbol{a}, \boldsymbol{b}, \boldsymbol{c}$, and $\boldsymbol{d}$ are independent) with $\boldsymbol{s}$ and $\boldsymbol{t}$ replacing $\boldsymbol{c}$ and $\boldsymbol{d}$. Consequently, (48) and (50) hold valid for $\tau$ after replacing $\sigma_{c}$ with the $\sigma_{s}$ in (52). This substitution reproduces (49) and (51) as it should.

\section{APPENDIX B}

\section{POLAR FORMS FOR $\boldsymbol{\xi}, \boldsymbol{\tau}, \boldsymbol{\chi}$, AND $\boldsymbol{\nu}$}

In this section, we derive the polar forms of $\xi, \tau, \chi$, and $\nu$ and demonstrate their use in determining the joint pdf relating $\boldsymbol{\nu}$ and $\boldsymbol{\xi}$. Consider the constituent components of (10): $\boldsymbol{\tau}=$ $(\boldsymbol{a} f-b e) /\left(a^{2}+b^{2}\right)=(a t-b s) /\left(a^{2}+b^{2}\right)($ see Appendix B) and $\nu=\xi \chi=(\boldsymbol{a d}-\boldsymbol{d} \boldsymbol{c}) /\left(\boldsymbol{a}^{2}+\boldsymbol{b}^{2} \boldsymbol{a c}+\boldsymbol{b d}\right) /\left(\boldsymbol{a}^{2}+\boldsymbol{b}^{2}\right)$, where $\boldsymbol{\xi}=(\boldsymbol{a d}-\boldsymbol{d c}) /\left(\boldsymbol{a}^{2}+\boldsymbol{b}^{2}\right)$ and $\boldsymbol{\chi}=(\boldsymbol{a} \boldsymbol{c}+\boldsymbol{b} \boldsymbol{d}) /\left(\boldsymbol{a}^{2}+\boldsymbol{b}^{2}\right)$. We begin by converting each into polar coordinates, setting $a=$ $\boldsymbol{r}_{\mathbf{1}} \cos \phi_{\mathbf{1}}, \boldsymbol{b}=\boldsymbol{r}_{\mathbf{1}} \sin \phi_{\mathbf{1}}, \boldsymbol{c}=\boldsymbol{r}_{\mathbf{2}} \cos \phi_{2}, \boldsymbol{d}=\boldsymbol{r}_{\mathbf{2}} \sin \phi_{\mathbf{2}}$ $c=r_{3} \cos \phi_{3}$, and $d=r_{3} \sin \phi_{3}$. The random variables $r_{1}$, $\boldsymbol{r}_{\mathbf{2}}$, and $\boldsymbol{r}_{3}$ have Rayleigh distributions while $\phi_{1}, \phi_{2}$, and $\phi_{3}$ are distributed uniformly over the interval $[-\pi: \pi]$ [25]. All the random variables are independent of each other. In polar polar coordinates, similar to those presented in [23], we have

$$
\begin{aligned}
& \xi=\frac{r_{\mathbf{2}}}{\boldsymbol{r}_{\mathbf{1}}} \sin \left(\phi_{\mathbf{2}}-\phi_{\mathbf{1}}\right)=\boldsymbol{R}_{\mathbf{1}} \sin \boldsymbol{\Phi}_{\mathbf{1}} \\
& \chi=\frac{\boldsymbol{r}_{\mathbf{2}}}{\boldsymbol{r}_{\mathbf{1}}} \cos \left(\phi_{\mathbf{2}}-\phi_{\mathbf{1}}\right)=\boldsymbol{R}_{\mathbf{1}} \cos \boldsymbol{\Phi}_{\mathbf{1}} \\
& \boldsymbol{\tau}=\frac{\boldsymbol{r}_{\mathbf{3}}}{\boldsymbol{r}_{\mathbf{1}}} \sin \left(\boldsymbol{\phi}_{\mathbf{3}}-\boldsymbol{\phi}_{\mathbf{1}}\right)=\boldsymbol{R}_{\mathbf{2}} \sin \boldsymbol{\Phi}_{\mathbf{2}} \\
& \boldsymbol{\nu}=\boldsymbol{\xi} \chi=\boldsymbol{R}_{\mathbf{1}}^{2} \sin \boldsymbol{\Phi}_{\mathbf{1}} \cos \boldsymbol{\Phi}_{\mathbf{1}}
\end{aligned}
$$

where $R_{1}=r_{2} / r_{1}, R_{2}=r_{3} / r_{1}, \Phi_{1}=\phi_{2}-\phi_{1}$, and $\Phi_{2}=\phi_{3}-\phi_{1} . \Phi_{1}$ and $\Phi_{2}$ are uniformly distributed over $[-\pi: \pi]$ and are independent of $\boldsymbol{R}_{\mathbf{1}}, \boldsymbol{R}_{\mathbf{2}}$, and each other. The joint distribution relating $\boldsymbol{r}_{\mathbf{1}}, \boldsymbol{r}_{\mathbf{2}}$, and $\boldsymbol{r}_{\mathbf{3}}$ is

$$
\begin{aligned}
f\left(r_{1}, r_{2}, r_{3}\right) & =f\left(r_{1}\right) f\left(r_{2}\right) f\left(r_{3}\right) \\
& =\frac{r_{1} r_{2} r_{3}}{\sigma_{a}^{2} \sigma_{c}^{2} \sigma_{s}^{2}} e^{-r_{1}^{2} / 2 \sigma_{a}^{2}} e^{-r_{2}^{2} 2 \sigma_{c}^{2}} e^{-r_{3}^{2} / 2 \sigma_{s}^{2}} .
\end{aligned}
$$

For a more general form of the pdf relating $\boldsymbol{r}_{\mathbf{1}}, \boldsymbol{r}_{\mathbf{2}}, \boldsymbol{\Phi}_{\mathbf{1}}$, and $\boldsymbol{\Phi}_{\mathbf{2}}$ that considers additional dependencies see [23]. To find the joint pdf relating relating $\boldsymbol{R}_{\mathbf{1}}$ and $\boldsymbol{R}_{\mathbf{2}}$, begin by letting $\boldsymbol{R}_{\mathbf{1}}=\boldsymbol{r}_{\mathbf{2}} / \boldsymbol{r}_{\mathbf{1}}$, $R_{2}=r_{3} / r_{1}$, and $z=r_{1}$. The solutions to these equations are $r_{1}=z, r_{2}=z R_{1}$, and $r_{3}=z R_{2}$. The Jacobian for the transformation is $z^{2}$. Substituting these solutions into (58) and integrating with respect to $z$, we have

$$
\begin{aligned}
f(R 1, R 2) & =\frac{R_{1} R_{2}}{\sigma_{a}^{2} \sigma_{c}^{2} \sigma_{s}^{2}} \int_{0}^{\infty} z^{5} e^{-z^{2}\left(1 / 2 \sigma_{a}^{2}+R_{1}^{2} / 2 \sigma_{c}^{2}+R_{2}^{2} / 2 \sigma_{s}^{2}\right)} \\
& =\frac{R_{1} R_{2}}{\sigma_{a}^{2} \sigma_{c}^{2} \sigma_{s}^{2}}\left(\frac{1}{2 \sigma_{a}^{2}}+\frac{R_{1}^{2}}{2 \sigma_{c}^{2}}+\frac{R_{2}^{2}}{2 \sigma_{s}^{2}}\right)^{-1}
\end{aligned}
$$




$$
=8 R_{1} R_{2} \frac{\sigma_{s}^{4}}{\sigma_{a}^{2} \sigma_{c}^{2}}\left(\frac{\sigma_{s}^{2}}{\sigma_{c}^{2}}\left[\frac{\sigma_{c}^{2}}{\sigma_{a}^{2}}+R_{1}^{2}\right]+R_{2}^{2}\right)^{-1}
$$

Integrating out $R_{2}$ and $R_{1}$ we arrive at the following marginal distributions:

$$
\begin{aligned}
& f\left(R_{1}\right)=\frac{2 \frac{\sigma_{c}^{2}}{\sigma_{a}^{2}} R_{1}}{\left(\frac{\sigma_{c}^{2}}{\sigma_{a}^{2}}+R_{1}^{2}\right)^{2}} \\
& f\left(R_{2}\right)=\frac{\frac{2 \sigma_{s}^{2}}{\sigma_{a}^{2} R_{2}}}{\left(\frac{\sigma_{s}^{2}}{\sigma_{a}^{2}+R_{2}^{2}}\right)^{2}} .
\end{aligned}
$$

For a moment we return our attention to $\boldsymbol{\nu}$. From (60), we can easily show that $\mathrm{E}\left[\boldsymbol{R}_{\mathbf{1}}^{2}\right]$ is not finite. As a consequence, $\mathrm{E}[|\boldsymbol{\nu}|]=$ $\mathrm{E}\left[\boldsymbol{R}_{\mathbf{1}}^{2}\right] \mathrm{E}\left[\left|\cos \boldsymbol{\Phi}_{\mathbf{1}} \sin \boldsymbol{\Phi}_{\mathbf{1}}\right|\right]$ is also infinite as is the variance of $\boldsymbol{\nu}$.

We can now find the joint distribution $f(\nu, \xi)$. Remembering that $\xi=R_{1} \sin \Phi_{1}$ and $\nu=R_{1}^{2} \cos \Phi_{1} \sin \Phi_{1}$, we can find the expression for $f(\nu, \xi)$ by substituting the solutions $\Phi_{1}=\cos ^{-1}(\xi / \nu)$ and $R_{1}=\xi \sqrt{\nu^{2}+\xi^{4}}$ (with Jacobian $\left.J=\xi^{2} \sqrt{\nu^{2}+\xi^{4}}\right)$ into $f\left(R_{1}, \Phi_{1}\right)=f\left(R_{1}\right) f\left(\Phi_{1}\right)$

$$
f(\nu, \xi)=\frac{\frac{\sigma_{c}^{2}}{\sigma_{a}^{2}} \xi^{3}}{\pi\left(\xi^{2}\left[\frac{\sigma_{c}^{2}}{\sigma_{a}^{2}}+\xi^{2}\right]+\nu^{2}\right)^{2}} .
$$

\section{APPENDIX C \\ CONDITIONAL PDF DERIVATIONS}

In this section, we analyze the effects of the constraints posed in (11) and (13) on the random variables $\boldsymbol{\xi}, \boldsymbol{\tau}$, and $\boldsymbol{\nu}$. The constraint shown in (11) restricts the possible values of $\boldsymbol{\xi}$. In order to quantify the influence of this constraint, we will find expressions for $\mathrm{E}\left[|\boldsymbol{\xi}||| \xi \mid<\rho_{1}\right], \mathrm{E}\left[|\boldsymbol{\tau}||| \xi \mid<\rho_{1}\right]$, and $\mathrm{E}\left[|\boldsymbol{\nu}||| \xi \mid<\rho_{1}\right]$. First, we note that

$$
\begin{aligned}
\mathrm{E}[|\cdot||| \xi \mid<\rho] & =\frac{\int_{-\rho}^{\rho} \mathrm{E}[|\cdot| \mid \xi] f(\xi) d \xi}{\int_{-\rho}^{\rho} f(\xi) d \xi} \\
& =\frac{1}{\rho} \frac{\sigma_{c}^{2}}{\sigma_{a}^{2}}\left[\frac{\sigma_{c}^{2}}{\sigma_{a}^{2}}+\rho^{2}\right]^{1 / 2} \int_{-\rho}^{\rho} \mathrm{E}[|\cdot| \mid \xi] f(\xi) d \xi .
\end{aligned}
$$

The initial step is to find $\mathrm{E}[|\xi| \mid \xi], \mathrm{E}[|\boldsymbol{\tau}| \mid \xi]$, and $\mathrm{E}[|\boldsymbol{\nu}| \mid \xi]$. Obviously, we have

$$
\mathrm{E}[|\xi| \mid \xi]=\xi
$$

$$
\begin{aligned}
\mathrm{E}\left[|\boldsymbol{\xi}||\xi|<\rho_{1}\right] & =\frac{\int_{-\rho_{1}}^{\rho_{1}} E[|\boldsymbol{\xi}| \mid \xi] f(\xi) d \xi}{\int_{-\rho_{1}}^{\rho_{1}} f(\xi) d \xi} \\
& =\frac{1}{\rho_{1}} \frac{\sigma_{c}^{2}}{\sigma_{a}^{2}}\left[\frac{\sigma_{c}^{2}}{\sigma_{a}^{2}}+\rho_{1}^{2}\right]^{1 / 2} \frac{4}{\pi} \int_{0}^{\rho_{1}} \frac{\frac{\sigma_{c}^{2}}{\sigma_{a}^{2}} \xi}{2\left[\frac{\sigma_{c}^{2}}{\sigma_{a}^{2}}+\xi^{2}\right]^{3 / 2}} d \xi \\
& =\frac{1}{\rho_{1}}\left(\frac{\sigma_{c}}{\sigma_{a}}\left[\frac{\sigma_{c}^{2}}{\sigma_{a}^{2}}+\rho_{1}^{2}\right]^{1 / 2}-\frac{\sigma_{c}^{2}}{\sigma_{a}^{2}}\right) \\
\mathrm{E}\left[|\boldsymbol{\tau}||| \xi \mid<\rho_{1}\right] & =\frac{\int_{-\rho_{1}}^{\rho_{1}} E[|\boldsymbol{\tau}| \mid \xi] f(\xi) d \xi}{\int_{-\rho_{1}}^{\rho_{1}} f(\xi) d \xi} \\
& =\frac{1}{\rho_{1}} \frac{\sigma_{c}^{2}}{\sigma_{a}^{2}}\left[\frac{\sigma_{c}^{2}}{\sigma_{a}^{2}}+\rho_{1}^{2}\right]^{1 / 2} \frac{4}{\pi} \int_{0}^{\rho_{1}} \frac{\left(\frac{|K|}{\sigma_{a}^{2} \sigma_{c}^{4}}\left[\frac{\sigma_{c}^{2}}{\sigma_{a}^{2}}+\xi^{2}\right]\right)^{1 / 2} \frac{\sigma_{c}^{2}}{\sigma_{a}^{2}}}{2\left[\frac{\sigma_{c}^{2}}{\sigma_{a}^{2}}+\xi^{2}\right]^{3 / 2}} d \xi \\
& =\frac{2}{\pi \rho_{1}}\left(\frac{|K|}{\sigma_{a}^{4} \sigma_{c}^{2}}\right)^{1 / 2}\left(\frac{\sigma_{c}^{2}}{\sigma_{a}^{2}}+\rho_{1}^{2}\right)^{1 / 2} \frac{\tan ^{-1}\left(\frac{\sigma_{a}}{\sigma_{c}} \rho_{1}\right)}{\mathrm{E}\left[|\boldsymbol{\nu}||| \xi \mid<\rho_{1}\right]}=\frac{\int_{-\rho_{1}}^{\rho_{1}} E[|\boldsymbol{\nu}| \mid \xi] f(\xi) d \xi}{\int_{-\rho_{1}}^{\rho_{1}} f(\xi) d \xi} \\
& =\frac{1}{\rho_{1}} \frac{\sigma_{c}^{2}}{\sigma_{a}^{2}}\left[\frac{\sigma_{c}^{2}}{\sigma_{a}^{2}}+\rho_{1}^{2}\right]^{1 / 2} \frac{4}{\pi} \int_{0}^{\rho_{1}} \frac{\left(\xi^{2}\left[\frac{\sigma_{c}^{2}}{\sigma_{a}^{2}}+\xi^{2}\right]\right)^{1 / 2} \frac{\sigma_{c}^{2}}{\sigma_{a}^{2}}}{2\left[\frac{\sigma_{c}^{2}}{\sigma_{a}^{2}}+\xi^{2}\right]^{3 / 2}} d \xi \\
& =\frac{1}{\pi \rho_{1}} \frac{\sigma_{c}^{2}}{\sigma_{a}^{2}}\left(\frac{\sigma_{a}^{2}}{\sigma_{c}^{2}}+\rho_{1}^{2}\right)^{1 / 2} \ln \left(1+\frac{\sigma_{c}^{2}}{\sigma_{a}^{2}} \rho_{1}^{2}\right) \cdot
\end{aligned}
$$




$$
\begin{aligned}
& \mathrm{E}\left[|\boldsymbol{\xi}||| R_{1} \mid<\rho_{3}\right]=\frac{\int_{-\pi}^{\pi} \int_{0}^{\rho_{3}} \xi f\left(R_{1}\right) f\left(\Phi_{1}\right) d R_{1} d \Phi_{1}}{\int_{0}^{\rho_{3}} f\left(R_{1}\right) d R_{1}} \\
& =\frac{1}{\rho_{3}^{2}}\left(\frac{\sigma_{c}^{2}}{\sigma_{a}^{2}}+\rho_{3}^{2}\right) \int_{-\pi}^{\pi} \int_{0}^{\rho_{3}} \frac{R_{1}^{2}\left|\sin \Phi_{1}\right| \frac{\sigma_{c}^{2}}{\sigma_{a}^{2}}}{\pi\left(\frac{\sigma_{c}^{2}}{\sigma_{a}^{2}}+r^{2}\right)^{2}} d R_{1} d \Phi_{1} \\
& =\frac{2}{\pi \rho_{3}} \frac{\sigma_{c}^{2}}{\sigma_{a}^{2}}\left(\frac{1}{\rho} \frac{\sigma_{a}}{\sigma_{c}} \tan ^{-1}\left[\frac{\sigma_{a}}{\sigma_{c}} \rho_{3}\right]\left[\frac{\sigma_{c}^{2}}{\sigma_{a}^{2}}+\rho_{3}^{2}\right]-1\right) . \\
& \mathrm{E}\left[|\boldsymbol{\tau}||| R_{1} \mid<\rho_{3}\right] \\
& =\frac{\int_{-\pi}^{\pi} \int_{0}^{\rho_{3}} \int_{0}^{\infty} \tau f\left(R_{1}, R_{2}\right) f\left(\Phi_{2}\right) d R_{2} d R_{1} d \Phi_{2}}{\int_{0}^{\rho_{3}} f\left(R_{1}\right) d R_{1}} \\
& =\frac{1}{\rho_{3}^{2}}\left(\frac{\sigma_{c}^{2}}{\sigma_{a}^{2}}+\rho_{3}^{2}\right) \\
& \times \int_{-\pi}^{\pi} \int_{0}^{\rho_{3}} \int_{0}^{\infty} \frac{\sigma_{s}^{4}}{\sigma_{a}^{2} \sigma_{c}^{2}} \frac{8 R_{1} R_{2}^{2}\left|\sin \Phi_{1}\right|}{\frac{\sigma_{s}^{2}}{\sigma_{c}^{2}}\left[\frac{\sigma_{c}^{2}}{\sigma_{a}^{2}}+R_{1}^{2}\right]+R_{2}^{2}} d R_{2} d R_{1} d \Phi_{2} \\
& =\frac{1}{\rho_{3}^{2}} \frac{\sigma_{c}}{\sigma_{a}}\left[\frac{|K|}{\sigma_{a}^{4} \sigma_{c}^{2}}\left(\frac{\sigma_{c}^{2}}{\sigma_{a}^{2}}+\rho_{3}^{2}\right)\left(\frac{\sigma_{a}}{\sigma_{b}}\left[\frac{\sigma_{c}^{2}}{\sigma_{a}^{2}}+\rho_{3}^{2}\right]-1\right)\right]^{1 / 2} \\
& \mathrm{E}\left[|\boldsymbol{\nu}||| R_{1} \mid<\rho_{3}\right]=\frac{\int_{-\pi}^{\pi} \int_{0}^{\rho_{3}} \nu f\left(R_{1}\right) f\left(\Phi_{1}\right) d r d \Phi_{1}}{\int_{0}^{\rho_{3}} f\left(R_{1}\right) d R_{1}} \\
& =\frac{\frac{\sigma_{c}^{2}}{\sigma_{a}^{2}}+\rho_{3}^{2}}{\rho_{3}^{2}} \int_{-\pi}^{\pi} \int_{0}^{\rho_{3}} \frac{R_{1}^{3}\left|\cos \Phi_{1} \sin \Phi_{1}\right| \frac{\sigma_{c}^{2}}{\sigma_{a}^{2}}}{\pi\left(\frac{\sigma_{c}^{2}}{\sigma_{a}^{2}}+r^{2}\right)^{2}} d R_{1} d \Phi_{1} \\
& =\frac{\frac{\sigma_{c}^{2}}{\sigma_{a}^{2}}}{\pi}\left[\frac{\frac{\sigma_{c}^{2}}{\sigma_{a}^{2}}+\rho_{3}^{2}}{\rho_{3}^{2}} \ln \left(\frac{\frac{\sigma_{c}^{2}}{\sigma_{a}^{2}}+\rho_{3}^{2}}{\frac{\sigma_{c}^{2}}{\sigma_{a}^{2}}}\right)-1\right]
\end{aligned}
$$

To determine the other expected values, we first determine the conditional distribution functions of $\boldsymbol{\xi}$ and $\boldsymbol{\nu}$ with respect to $\boldsymbol{\xi}$

$$
\begin{gathered}
f(\tau \mid \xi)=\frac{f(\xi, \tau)}{f(\xi)}=\frac{2\left(\frac{|K|}{\sigma_{a}^{2} \sigma_{c}^{4}}\left[\frac{\sigma_{c}^{2}}{\sigma_{a}^{2}}+\xi^{2}\right]\right)^{3 / 2}}{\pi\left(\frac{|K|}{\sigma_{a}^{2} \sigma_{c}^{4}}\left[\frac{\sigma_{c}^{2}}{\sigma_{a}^{2}}+\xi^{2}\right]+\tau^{2}\right)^{2}} \\
f(\nu \mid \xi)=\frac{f(\xi, \nu)}{f(\xi)}=\frac{2\left(\xi^{2}\left[\frac{\sigma_{c}^{2}}{\sigma_{a}^{2}}+\xi^{2}\right]\right)^{3 / 2}}{\pi\left(\xi^{2}\left[\frac{\sigma_{c}^{2}}{\sigma_{a}^{2}}+\xi^{2}\right]+\nu^{2}\right)^{2}}
\end{gathered}
$$

Using the fact that $\int_{-\infty}^{\infty} 2 t_{o}^{3 / 2}|t| / \pi\left(t_{o}+t^{2}\right)^{2} d t=2 / \pi \sqrt{t_{o}}$, we find

$$
\begin{aligned}
& \mathrm{E}[|\boldsymbol{\tau}| \mid \xi]=\frac{2}{\pi}\left(\frac{|K|}{\sigma_{a}^{2} \sigma_{c}^{4}}\left[\frac{\sigma_{c}^{2}}{\sigma_{a}^{2}}+\xi^{2}\right]\right)^{1 / 2} \\
& \mathrm{E}[|\boldsymbol{\nu}| \mid \xi]=\frac{2}{\pi}\left(\xi^{2}\left[\frac{\sigma_{c}^{2}}{\sigma_{a}^{2}}+\xi^{2}\right]\right)^{1 / 2} .
\end{aligned}
$$

Inserting (64), (67) and (68) into (63) we have (69)-(71), shown at the bottom of the previous page.

Finally, we quantify the influence of constraint (13) on $\boldsymbol{\xi}$, $\boldsymbol{\tau}, \boldsymbol{\nu}$ by evaluating $\mathrm{E}\left[|\boldsymbol{\xi}||| R_{1} \mid<\rho_{3}\right], \mathrm{E}\left[|\boldsymbol{\tau}||| R_{1} \mid<\rho_{3}\right]$, and
$\mathrm{E}\left[|\nu||| R_{1} \mid<\rho_{3}\right]$, where $R_{1}=\sqrt{\xi^{2}+\chi^{2}}$. We find the expected values in a slightly different manner than that shown in (63). In Appendix B, we discussed how $\boldsymbol{\xi}, \boldsymbol{\tau}, \boldsymbol{\nu}$ can be expressed as functions of the random variables $\boldsymbol{R}_{\mathbf{1}}, \boldsymbol{R}_{\mathbf{2}}, \boldsymbol{\Phi}_{\mathbf{1}}$, and $\boldsymbol{\Phi}_{\mathbf{2}}$ [see (54), (56), and (57)]. Therefore, we can use the pdfs in (59) and (60) to calculate the expected value as in (72)-(74), shown at the top of the page.

\section{ACKNOWLEDGMENT}

The authors would like to thank their outstanding reviewers who helped to improve both the quality and readability of this work.

\section{REFERENCES}

[1] J. Magarey and A. Dick, "Multiresolution stereo image matching using complex wavelets," in Proc. 14th Int. Conf. Pattern Recognition, Aug. 1998, vol. 1, pp. 4-7.

[2] D. Fleet, "Disparity from local weighted phase-correlation," in Proc. IEEE Int. Conf. Systems, Man and Cybernetics, 1994, vol. 1, pp. 48-54 [Online]. Available: http://dx.doi.org/10.1109/ICSMC.1994.399810

[3] D. Papadimitriou and T. Dennis, "Stereo disparity analysis using phase correlation," Electron. Lett., vol. 30, no. 18, pp. 1475-1477, 1994.

[4] J. Weng, "Windowed fourier phase. Completeness and signal reconstruction," IEEE Trans. Signal Process., vol. 41, no. 2, pp. 657-666, Feb. 1993.

[5] F. Solari, S. Sabatini, and G. Bisio, "Fast technique for phase-based disparity estimation with no explicit calculation of phase," Electron. Lett., vol. 37, no. 23, pp. 1382-1383, 2001. 
[6] T. Frohlinghaus and J. Buhmann, "Regularizing phase-based stereo," in Proc. 13th Int. Conf. Pattern Recognition, Aug. 25-29, 1996, vol. 1, pp. 451-455.

[7] M. Hansen, K. Daniilidis, and G. Sommer, "Optimization of stereo disparity estimation using the instantaneous frequency," Comput. Anal. Images Patterns, pp. 321-328, 1997.

[8] A. C. Bovik, The Handbook of Image \& Video Processing. San Diego, CA: Academic, 2000.

[9] T.-Y. Chen, A. C. Bovik, and L. K. Cormack, "Stereoscopic ranging by matching image modulations," IEEE Trans. Image Process., vol. 8 , no. 6, pp. 785-797, Jun. 1999.

[10] M. Jenkin and A. Jepson, "Recovering local surface structure through local phase difference measurements," Comput. Vis., Graph., Image Process.: Image Understand., vol. 59, no. 1, pp. 72-93, 1994.

[11] A. D. Jepson and M. Jenkin, "Fast computation of disparity from phase differences," in Proc. IEEE Computer Vision and Pattern Recognition, 1989 , pp. 398-403.

[12] T. Sanger, "Stereo disparity computation using gabor filters," Biol. Cybern., vol. 59, pp. 405-418, 1988.

[13] Y.Xu, J. Zhou, and G. Zhai, "2D phase-based matching in uncalibrated images," in Proc. IEEE Workshop on Signal Processing Systems Design and Implementation, 2005, pp. 325-330.

[14] C.-J. Westelius, "Focus of Attention and Gaze Control for Robot Vision,” Ph.D. dissertation, Linköping Univ., Linköping, Sweden, 1995.

[15] R. Wilson and H. Knutsson, "A multiresolution stereopsis algorithm based on the Gabor representation," presented at the 3rd Int. Conf. Image Processing and its Applications, 1989.

[16] U. R. Dhond and J. Aggarwal, "Structure from stereo-A review," IEEE Trans. Syst., Man, Cybern., vol. 19, no. 6, pp. 1489-1510, Nov. -Dec. 1989.

[17] A. Cozzi, B. Crespi, F. Valentinotti, and F. Woergoetter, "Performance of phase-based algorithms for disparity estimation," Mach. Vis. Appl., vol. 9, no. 5-6, pp. 334-340, 1997.

[18] D. Fleet and A. Jepson, "Stability of phase information," IEEE Trans. Pattern Anal. Mach. Intell., vol. 15, no. 12, pp. 1253-1268, Dec. 1993.

[19] L.-D. Cai and J. Mayhew, "Note on some phase differencing algorithms for disparity estimation," Int. J. Comput. Vis., vol. 22, no. 2, pp. 111-124, 1997.

[20] A. Jepson and D. Fleet, "Phase singularities in scale-space," Image Vis. Comput., vol. 9, no. 5, pp. 338-343, 1991.

[21] M. Ouali, D. Ziou, and C. Laurgeau, "Inaccurate phase-based disparities removal," in Proc. Int. Conf. Electronics, Circuits, and Systems, Sep. 1999, vol. 2, pp. 5-8.

[22] D. Fleet, A. Jepson, and M. Jenkin, "Phase-based disparity measurement," Comput. Vis., Graph., Image Process.: Image Understand., vol. 53, no. 2, pp. 198-210, 1991.

[23] D. Fleet, Measurement of Image Velocity. Norwell, MA: Kluwer, 1992.

[24] A. Papoulis, Probability, Random Variables, and Stochastic Processes. New York: McGraw-Hill, 1965.

[25] D. Gabor, "Theory of communication," J. Inst. Elect. Eng., vol. 93, pp. 429-441, 1946.

[26] Q.-S. Chen and F. Deconinck, "Foveal-view stereopsis using symmetric phase-only matched filtering," Proc. SPIE, vol. 2233, pp. 46-53, 1994.

[27] T.-Y. Chen and A. C. Bovik, "Stereo vision using gabor wavelets," in Proc. IEEE Southwest Symp. Image Analysis and Interpretation, Apr. 1994, pp. 13-17.

[28] M. Clark, A. C. Bovik, and W. Geisler, "Texture segmentation using a class of narrowband filters," in Proc. IEEE Int. Conf. Acoustics, Speech, and Signal Processing, Apr. 1987, vol. 12, pp. 571-574.

[29] M. Clark and A. C. Bovik, "Experiments in segmenting texton patterns using localized spatial filters," Pattern Recognit., vol. 22, no. 6, pp. 707-717, 1989.

[30] J. Jones and L. Palmer, "An evaluation of the two-dimensional gabor filter model of simple receptive fields in cat striate cortex," J. Neurophysiology, vol. 58, pp. 1233-1258, 1987.

[31] A. Anzai, I. Ohzawa, and R. Freeman, "Neural mechanisms for processing binocular information I. Simple cells," J. Neurophysiology, vol. 82, no. 2, pp. 891-908, Aug. 1999.

[32] M. Clark and A. C. Bovik, "Texture discrimination using a model of the visual cortex," in Proc. IEEE Int. Conf. Systems, Man, and Cybernetics, 1986, pp. $1425-1430$.

[33] B. Crespi and G. Tecchiolli, "Adaptive gabor filters for phase-based disparity estimation," Int. J. Pattern Recognit. Artif. Intell., vol. 13, no. 5, pp. 591-614, 1999.

[34] D. J. Field, "Relations between the statistics of natural images and the response properties of cortical cells," J. Opt. Soc. Amer. A, vol. 4, no. 12, pp. 2379-2379, 1987.
[35] Z. Wang and E. S. Bovik, "Multiscale structural similarity for image quality assessment," in Proc. Conf. Rec. 37th Asilomar Conf. Signals, Systems and Computers, Nov. 9-12, 2003, vol. 2, pp. 1398-1402.

[36] Z. Wang, A. Bovik, H. Sheikh, and E. Simoncelli, "Image quality assessment: From error visibility to structural similarity," IEEE Trans. Image Process., vol. 13, no. 4, pp. 600-612, Apr. 2004.

[37] P. R. Rider, "Generalized cauchy distributions," Ann. Inst. Statist. Math., vol. 9, pp. 215-223, 1957.

[38] J. H. Miller and J. B. Thomas, "Detectors for discrete-time signals in non-gaussian noise," IEEE Trans. Inf. Theory, vol. IT-18, no. 2, pp. 241-250, Mar. 1972.

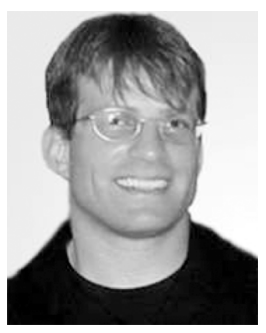

James Peter Monaco (S'02-M'07) received the B.S. degree in computer engineering and the M.S. degree in electrical engineering from Texas A\&M University, College, Station, in 1996 and 1998, respectively, and the Ph.D. degree in electrical engineering from the University of Texas at Austin in 2007.

He has worked for Texas Instruments, Inc. and Raytheon Corporation, designing infrared tracking systems for military aircraft. He also spent six years working for VuCOMP, a medical imaging company dedicated to the automatic detection of disease. He is currently a member of the Biomedical Engineering Department, Rutgers University, Piscataway, NJ.

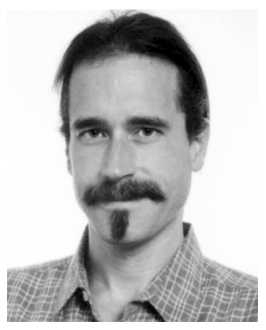

Alan Conrad Bovik (S'80-M'81-SM'89-F'96) received the B.S., M.S., and Ph.D. degrees in electrical and computer engineering from the University of Illinois at Urbana-Champaign, Urbana, in 1980, 1982, and 1984 , respectively.

He is currently the Curry/Cullen Trust Endowed Professor at The University of Texas at Austin, where he is the Director of the Laboratory for Image and Video Engineering (LIVE) in the Center for Perceptual Systems. His research interests include image and video processing, computational vision, digital microscopy, and modeling of biological visual perception. He has published over 450 technical articles in these areas and holds two U.S. patents. He is also the author of The Handbook of Image and Video Processing (Elsevier, 2005, 2nd ed.) and Modern Image Quality Assessment (Morgan \& Claypool, 2006)

Dr. Bovik has received a number of major awards from the IEEE Signal Processing Society, including: the Education Award (2007); the Technical Achievement Award (2005), the Distinguished Lecturer Award (2000); and the Meritorious Service Award (1998). He is also a recipient of the Distinguished Alumni Award from the University of Illinois at Urbana-Champaign (2008), the IEEE Third Millennium Medal (2000), and two journal paper awards from the international Pattern Recognition Society (1988 and 1993). He is a Fellow of the Optical Society of America and a Fellow of the Society of Photo-Optical and Instrumentation Engineers. He has been involved in numerous professional society activities, including: Board of Governors, IEEE Signal Processing Society, 1996-1998; Editor-in-Chief, IEEE TranSACTIONS ON IMAGE PROCESSING, 1996-2002; Editorial Board, PROCEEDINGS OF THE IEEE, 1998-2004; Series Editor for Image, Video, and Multimedia Processing, Morgan and Claypool Publishing Company, 2003-present; and Founding General Chairman, First IEEE International Conference on Image Processing, Austin, TX, November 1994. He is a registered Professional Engineer in the State of Texas and is a frequent consultant to legal, industrial, and academic institutions.

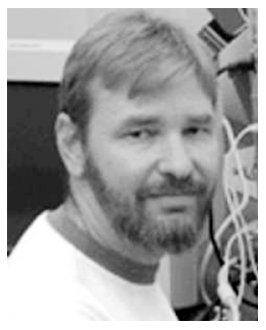

Lawrence K. Cormack received the B.S. degree from the University of Florida, Gainesville, and the Ph.D. degree in physiological optics from the University of California, Berkeley, in 1992.

$\mathrm{He}$ is currently an Associate Professor of psychology and neuroscience at the University of Texas (UT) at Austin, an Adjunct Associate Professor of vision science at the University of Houston, Houston, $\mathrm{TX}$, and an active member of the UT Center for Perceptual Systems. His early research interest was in contrast processing in stereoscopic vision, which is a good model system in which to study how the brain combines signals in general. More recently, his laboratory has been investigating the image properties that attract gaze when viewing natural scenes and when searching for targets imbedded in naturalistic background noise. 